\title{
Fast Solvers of Weakly Singular Integral Equations of the Second Kind
}

\section{Sumaira Rehman, Arvet Pedas and Gennadi Vainikko}

\author{
University of Tartu,Institute of Mathematics and Statistics \\ J.Liivi 2, Tartu, Estonia \\ E-mail: sumaira.rehman@ut.ee \\ E-mail(corresp.): arvet.pedas@ut.ee \\ E-mail: gennadi.vainikko@ut.ee
}

Received February 11, 2018; revised September 13, 2018; accepted September 13, 2018

\begin{abstract}
We discuss the bounds of fast solving weakly singular Fredholm integral equations of the second kind with a possible diagonal singularity of the kernel and certain boundary singularities of the derivatives of the free term when the information about the smooth coefficient functions in the kernel and about the free term is restricted to a given number of sample values. In this situation, a fast/quasifast solver is constructed. Thus the complexity of weakly singular integral equations occurs to be close to that of equations with smooth data without singularities. Our construction of fast/quasifast solvers is based on the periodization of the problem.
\end{abstract}

Keywords: complexity, fast solvers, weakly singular integral equations, Fredholm equations, periodization, trigonometric collocation.

AMS Subject Classification: 65Y20; 65T05; 45B05.

\section{Introduction}

Consider the weakly singular Fredholm integral equation of the second kind

$$
u(x)=\int_{0}^{1}\left(a(x, y)|x-y|^{-\nu}+b(x, y)\right) u(y) d y+f(x), \quad 0 \leq x \leq 1,
$$

where $0<\nu<1$. Denote by $C^{k}(\Omega)$ the set of $k$ times $(k \geq 0)$ continuously differentiable functions on a domain $\Omega$; in particular, $C=C[0,1]=C^{0}[0,1]$ denotes the Banach space of continuous functions $f$ on $[0,1]$ with the usual norm $\|f\|_{\infty}:=\max _{0 \leq x \leq 1}|f(x)|$. By $C^{m, \nu}=C^{m, \nu}(0,1)(m \in \mathbb{N}, 0<\nu<1)$ we 
denote the Banach space of functions $f \in C[0,1] \cap C^{m}(0,1)$ such that

$$
\|f\|_{C^{m, \nu}}:=\|f\|_{\infty}+\sum_{k=1}^{m} \sup _{0<x<1}(x(1-x))^{k-1+\nu}\left|f^{(k)}(x)\right|<\infty .
$$

For given $m \in \mathbb{N}:=\{1,2, \ldots\}$ and $\nu \in(0,1)$ we assume that

$$
f \in C^{m, \nu}(0,1) ; \quad(\mathrm{A} 2) \quad a, b \in C^{2 m}([0,1] \times[0,1]) ;
$$

the homogeneous equation $u(x)=\int_{0}^{1}\left(a(x, y)|x-y|^{-\nu}+b(x, y)\right) u(y) d y$, corresponding to (1.1) has in $C[0,1]$ only the trivial solution $u=0$.

Introduce also the following strengthened smoothness condition for functions $a$ and $b$ :

$$
a, b \in \mathcal{H}^{2 m, \mu}([0,1] \times[0,1]) \text { with a } \mu \in(0,1] .
$$

The Hoelder space $\mathcal{H}^{2 m, \mu}([0,1] \times[0,1])$ consists of functions $v \in C^{2 m}([0,1] \times$ $[0,1])$ with $\left(\frac{\partial}{\partial x}\right)^{i}\left(\frac{\partial}{\partial y}\right)^{j} v(x, y), i+j=2 m$, satisfying the Hoelder condition with the exponent $\mu \in(0,1]$ :

$$
\sup _{\substack{x_{1}, x_{2}, y_{1}, y_{2} \in[0,1],\left(x_{1}, y_{1}\right) \neq\left(x_{2}, y_{2}\right)}} \frac{\left|\left(\frac{\partial}{\partial x}\right)^{i}\left(\frac{\partial}{\partial y}\right)^{j} v\left(x_{1}, y_{1}\right)-\left(\frac{\partial}{\partial x}\right)^{i}\left(\frac{\partial}{\partial y}\right)^{j} v\left(x_{2}, y_{2}\right)\right|}{\left(\left|x_{2}-x_{1}\right|+\left|y_{2}-y_{1}\right|\right)^{\mu}}<\infty .
$$

Denote by $T=A+B$ the integral operator of equation (1.1) with operators $A$ and $B$ defined by

$$
(A u)(x)=\int_{0}^{1} a(x, y)|x-y|^{-\nu} u(y) d y, \quad(B u)(x)=\int_{0}^{1} b(x, y) u(y) d y .
$$

The operator $T: C[0,1] \rightarrow C[0,1]$ is compact; it is compact also as an operator from $C^{m, \nu}(0,1)$ into $C^{m, \nu}(0,1)$ (see [12]); thus, by Fredholm alternative, equation (1.1) has a solution $u \in C^{m, \nu}(0,1)$ which is unique in $C[0,1]$.

Our aim is to construct fast/quasifast solvers of equation (1.1). In a fast solver, the conditions of optimal accuracy and minimal arithmetical work are met. We mean the order optimality and order minimal work on a class of problems. In our case the class of problems is defined by the smoothness conditions which we set above on the free term and the kernel of equation (1.1), see (A1), (A2) or (A1), (A2'). More precisely, we use the following notions of fast/quasifast solvers.

Definition 1. By a $\left(C, C^{m, \nu}\right)$-fast solver of equation (1.1) we mean a solver which produces approximate solutions $u_{n} \in C[0,1], n \in \mathbb{N}$, such that

- given the values of $a, b$ and $f$, each at not more than $n_{*}$ points (depending on the solver, with $n_{*} \rightarrow \infty$ as $n \rightarrow \infty$ ), the parameters of $u_{n}$ can be determined at the cost of $\gamma_{m} n_{*}$ arithmetical operations and an accuracy

$$
\left\|u-u_{n}\right\|_{\infty}=\max _{0 \leq x \leq 1}\left|u(x)-u_{n}(x)\right| \leq c_{m} n_{*}^{-m}\|f\|_{C^{m, \nu}}
$$

is achieved, where $u$ is the solution of (1.1); 
- having the parameters of $u_{n}$ in hand, the value of $u_{n}$ at any point $x \in[0,1]$ is available at the cost of $\gamma_{m}^{\prime}$ arithmetical operations.

Here the constants $c_{m}, \gamma_{m}, \gamma_{m}^{\prime}$ are independent of $f$ and $n$.

Note that estimate (1.2) is information optimal even in the case where $a(x, y)=$ 0 for $x, y \in[0,1]$ (in this case the solution of (1.1) can be presented in the form $u=(I-B)^{-1} f$, where $I$ is the identity mapping and $(I-B)^{-1}$ is the inverse of the operator $I-B$ ). Namely (see original work [34] or lecture notes [29]), for any $m, m^{\prime} \in \mathbb{N}, \beta>0, \bar{\beta}>1$ and any solver of $(1.1)$ with $a(x, y) \equiv 0$ depending on $n_{*}$ evaluation points for $f \in C^{m}=C^{m}[0,1]$ and $b \in C^{m^{\prime}}([0,1] \times[0,1])$, there is a "bad" pair $f, b$ satisfying

$$
\|f\|_{C^{m}}:=\sum_{k=0}^{m}\left\|f^{(k)}\right\|_{\infty}=1, \quad\|b\|_{C^{m^{\prime}}([0,1] \times[0,1])} \leq \beta, \quad\left\|(I-B)^{-1}\right\|_{C \rightarrow C} \leq \bar{\beta},
$$

and such that, disregarding the amount of arithmetical work, the lower error bound

$$
\left\|u-u_{n}\right\|_{\infty} \geq c_{o} n_{*}^{-\min \left\{m, m^{\prime} / 2\right\}}
$$

holds where $c_{0}$ is a positive constant depending only on $m, m^{\prime}, \beta$ and $\bar{\beta}$. Thus, under traditional assumptions $f \in C^{m}[0,1], b \in C^{m}([0,1] \times[0,1])$, i.e. for $m^{\prime}=m$, only the accuracy order $\left\|u-u_{n}\right\|_{\infty} \leq c n_{\star}^{-m / 2}\|f\|_{C^{m}}$ can be achieved by any solver (this partial result has been established already in [4]). A further consequence of the lower error bound is that accuracy (1.2) is possible only if $m^{\prime} \geq 2 m$, and this explains the constellation of our assumption (A2) with $m^{\prime}=2 m$.

We speak about a $\left(L^{p}, C^{m, \nu}\right)$-fast solver $(1 \leq p<\infty)$ if the accuracy requirement (1.2) in Definition 1 is replaced by $\left\|u-u_{n}\right\|_{p} \leq c_{m} n_{\star}^{-m}\|f\|_{C^{m, \nu}}$, where $\|u\|_{p}=\left(\int_{0}^{1}|u(x)|^{p} d x\right)^{1 / p}$ is the standard norm of $u$ in $L^{p}(0,1)$. Similarly, we obtain a $\left(C, C^{m}\right)$-fast solver if in the accuracy requirement (1.2) the norm $\|f\|_{C^{m, \nu}}$ is replaced by $\|f\|_{C^{m}}$.

Definition 2. In a $\left(C, C^{m, \nu}\right)$-quasifast solver, the accuracy requirement (1.2) is replaced by

$$
\left\|u-u_{n}\right\|_{\infty} \leq c_{m} n_{\star}^{-m}\left(\log n_{\star}\right)\|f\|_{C^{m, \nu}},
$$

maintaining other requirements of Definition 1.

Remark 1. Estimate (1.2) can be rewritten with respect to the complexity

$$
n_{\star \star}:=\gamma_{m} n_{\star}
$$

of the solver in the equivalent form

$$
\left\|u-u_{n}\right\|_{\infty} \leq \bar{c}_{m} n_{\star \star}^{-m}\|f\|_{C^{m, \nu}}, \quad \bar{c}_{m}=\gamma_{m}^{m} c_{m} .
$$

This form of the estimate enables a comparison of $\left(C, C^{m, \nu}\right)$-fast solvers with different complexity parameters $\gamma_{m}$ : to smaller $\bar{c}_{m}$ there corresponds more effective solver. 
Remark 2. Consider also an alternative definition of a $\left(C, C^{m, \nu}\right)$-quasifast solver requiring accuracy (1.2) but allowing $\bar{\gamma}_{m} n_{\star} \log n_{\star}$ arithmetical operations. Then with respect to the complexity $n_{\star \star}=\bar{\gamma}_{m} n_{\star} \log n_{\star}$ estimate (1.2) takes the form

$$
\begin{aligned}
\left\|u-u_{n}\right\|_{\infty} & \leq c_{m}\left(\omega^{-1}\left(n_{\star \star} / \bar{\gamma}_{m}\right)\right)^{-m}\|f\|_{C^{m, \nu}} \\
& \sim c_{m} \bar{\gamma}_{m}^{m} n_{\star \star}^{-m}\left(\log \left(n_{\star \star} / \bar{\gamma}_{m}\right)\right)^{m}\|f\|_{C^{m, \nu}},
\end{aligned}
$$

where $\omega(x)=x \log x, \omega^{-1}(x) \sim x / \log x$ as $x \longrightarrow \infty$, i.e $\left(\omega^{-1}(x) \log x\right) / x \longrightarrow 1$ as $x \rightarrow \infty$. We see that for $m \geq 2$ Definition 2 is more restrictive (leads to a more high accuracy) than the alternative definition.

An intensive investigation of complexity for various kind of problems was started in $[22,23]$. In $[16,17,18,34]$ the complexity of the approximate solutions of Fredholm integral equations of the second kind with smooth kernels has been studied. Actually, also in $[28,30,31,33]$ fast or $\left(C, C^{m}\right)$-quasifast solvers have been constructed only for integral equations without singularities; for (1.1) this corresponds to the case $a(x, y) \equiv 0$. The construction of $\left(C, C^{m}\right)$-fast/quasifast solvers of periodic weakly singular integral equations of the second kind in case $a \not \equiv 0$ has been undertaken in [14]. Also some results of $[2,5,19,21]$ can be interpreted as a construction of $\left(L^{2}, C^{m}\right)$-fast/quasifast or $\left(L^{2}, W^{m, 2}\right)$-fast/quasifast solvers of periodic weakly singular integral equations ( $W^{m, 2}$ is the Sobolev space of functions on $(0,1)$, see Section 3.1). However, up to now, the construction of $\left(C, C^{m, \nu}\right)$-fast/quasifast solvers for nonperiodic equations (1.1) was an open problem. In the present paper we construct the solvers which under the conditions $\left(\mathrm{A} 1-(\mathrm{A} 3)\right.$ are $\left(C, C^{m, \nu}\right)$-quasifast and $\left(L^{p}, C^{m, \nu}\right)$-fast, $1 \leq p<\infty$. Moreover, these solvers are $\left(C, C^{m, \nu}\right)$-fast under the conditions (A1), (A2 $\left.2^{\prime}\right),(\mathrm{A} 3)$. Since we must discretize functions $a$ and $b$ of two variables, we use in our constructions $n_{\star}=O\left(n^{2}\right)$ that is more convenient rather than $n_{\star}=n$. Perhaps, for the numerical praxis the obtained solvers are of too complicated structure, so the results of the paper have mainly theoretical interest, they characterize the smallest possible complexity of discretization methods for weakly singular integral equations of the second kind. Thus we obtain a possibility to estimate how "good" an arbitrary discretization method is from the point of view of complexity.

We now outline the rest of the present paper. In Section 2 we introduce a change of variables and after that a change of unknown functions that reduce (1.1) into a periodic problem. Section 3 contains preliminaries mainly about the trigonometric interpolation. In Section 4 we adapt and modify the method of [14] for the periodized version of equation (1.1). After some preparation work (Section 5), in the course of Sections 6-7, we reorganize this method to obtain fast/quasifast solvers for (1.1). In Section 8 we shortly formulate some open problems which we hope to study in the future.

Throughout the paper $c, c^{\prime}, c_{1}, \ldots$ are some positive constants which can be different in different occurrences.

\section{Periodization of the integral equation}

It is well known $[6,12,26,32]$ (see also $[11,15,20]$ ) that the derivatives of a solution to a weakly singular integral equation such as (1.1), in general, have 
certain boundary singularities. With the help of a suitable change of variables $[8,10,13,25]$ (see also $[3,7]$ ) in the equation, these boundary singularities can be suppressed. Below we use a change integration variables and a change of the unknown functions to transform (1.1) into a 1-periodic problem.

To reduce (1.1) to a periodic form used in [14] we need a smooth function $g(t), 0<t<1$, which will be extended to 1-periodic function $\widetilde{g}$. For $\nu \in(0,1)$ and $m \in \mathbb{N}$, we introduce the function $g=g(t), \quad 0<t<1$, with

$$
g(t)= \begin{cases}t^{-\nu}, & 0<t \leq 1 / 3 \\ (1-t)^{-\nu}, & 2 / 3 \leq t<1 \\ \gamma(t), & 1 / 3<t<2 / 3\end{cases}
$$

where $\gamma(t)$ is a polynomial of degree $4 m+1$ determined by the conditions

$$
\gamma(t)^{(j)}(1 / 3)=\left.\left(\frac{d}{d t}\right)^{j} t^{-\nu}\right|_{t=1 / 3}, \quad \gamma(t)^{(j)}(2 / 3)=\left.\left(\frac{d}{d t}\right)^{j}(1-t)^{-\nu}\right|_{t=2 / 3}
$$

where $j=0,1, \ldots, 2 m$. After that we extend $g$ from $(0,1)$ into a 1-periodic function $\tilde{g} \in C^{2 m}(\mathbb{R} \backslash \mathbb{Z})$, where $\mathbb{R}:=(-\infty, \infty)$ and $\mathbb{Z}:=\{\ldots,-2,-1,0,1,2, \ldots\}$. Then (compare [14])

$$
\begin{aligned}
& \left|\widetilde{g}^{(j)}(t)\right| \leq c|t|^{-\nu-j}, \quad \text { for } \quad 0<|t| \leq 1 / 2, \quad j=0,1, \ldots, 2 m, \\
& \widetilde{g}(t)-|t|^{-\nu}=0, \quad \text { for } \quad|t| \leq 1 / 3 .
\end{aligned}
$$

Perform in equation (1.1) the change the variables $x=\varphi(t), y=\varphi(s)$, where $\varphi:[0,1] \longrightarrow[0,1]$ is given by the formula (see, e.g. $[8,13,25]$ )

$$
\varphi(t)=\frac{1}{c_{\star}} \int_{0}^{t} \sigma^{r-1}(1-\sigma)^{r-1} d \sigma, \quad c_{\star}=\int_{0}^{1} \sigma^{r-1}(1-\sigma)^{r-1} d \sigma=\frac{((r-1) !)^{2}}{(2 r-1) !}
$$

condition on the smoothing parameter $r \in \mathbb{N}$ will be set later. Clearly, $\varphi$ is a polynomial of degree $2 r-1: \varphi(t)=\frac{1}{c_{*}} \sum_{j=0}^{r-1}(-1)^{j}\left(\begin{array}{c}r-1 \\ j\end{array}\right) \frac{1}{r+j} t^{r+j}$. Moreover, we have $\varphi(0)=0, \varphi(1)=1, \varphi^{\prime}(t)>0$ for $0<t<1$. Since $(\varphi(t)-\varphi(s)) /(t-s)>0$ for $0 \leq t, s \leq 1, t \neq s$, equation (1.1) takes the form

$$
\begin{gathered}
u(\varphi(t))=\int_{0}^{1}\left[a(\varphi(t), \varphi(s))\left(\frac{\varphi(t)-\varphi(s)}{t-s}\right)^{-\nu}|t-s|^{-\nu}\right. \\
+b(\varphi(t), \varphi(s))] u(\varphi(s)) \varphi^{\prime}(s) d s+f(\varphi(t))
\end{gathered}
$$

or after some reorganizing,

$$
\bar{u}(t)=\int_{0}^{1}(\bar{a}(t, s) \widetilde{g}(t-s)+\bar{b}(t, s)) \bar{u}(s) d s+\bar{f}(t), \quad 0 \leq t \leq 1,
$$


where for $0 \leq t, s \leq 1, \bar{u}(t)=u(\varphi(t)), \bar{f}(t)=f(\varphi(t))$,

$$
\begin{aligned}
\bar{a}(t, s) & =a(\varphi(t), \varphi(s)) \Phi(t, s)^{-\nu} \varphi^{\prime}(s), \\
\bar{b}(t, s) & =\left[b(\varphi(t), \varphi(s))+a(\varphi(t), \varphi(s)) \Phi(t, s)^{-\nu}\left(|t-s|^{-\nu}-\widetilde{g}(t-s)\right)\right] \varphi^{\prime}(s), \\
\Phi(t, s) & =\left\{\begin{array}{cl}
(\varphi(t)-\varphi(s)) /(t-s), & t \neq s, \\
\varphi^{\prime}(t), & t=s .
\end{array}\right.
\end{aligned}
$$

Since $\varphi(t)$ is a polynomial of degree $2 r-1, \Phi(t, s)$ is a polynomial of degree $2 r-2$. Now we introduce a change of the unknown function. With respect to

$$
\widetilde{u}(t):=\varphi^{\prime}(t)^{(1-\nu) / 2} \bar{u}(t)=\varphi^{\prime}(t)^{(1-\nu) / 2} u(\varphi(t))
$$

equation (2.5) reads as

$$
\widetilde{u}(t)=\int_{0}^{1}(\widetilde{a}(t, s) \widetilde{g}(t-s)+\widetilde{b}(t, s)) \widetilde{u}(s) d s+\widetilde{f}(t), \quad 0 \leq t \leq 1,
$$

or

$$
\widetilde{u}=\widetilde{T} \widetilde{u}+\widetilde{f}, \quad \tilde{f}(t)=\varphi^{\prime}(t)^{(1-\nu) / 2} f(\varphi(t))
$$

where

$$
\widetilde{T}=\widetilde{A}+\widetilde{B}
$$

with $\widetilde{A}$ and $\widetilde{B}$ defined by

$$
\begin{gathered}
(\widetilde{A} u)(t)=\int_{0}^{1} \widetilde{a}(t, s) \widetilde{g}(t-s) u(s) d s, \quad(\widetilde{B} u)(t)=\int_{0}^{1} \widetilde{b}(t, s) u(s) d s \\
\widetilde{a}(t, s)=\varphi^{\prime}(t)^{(1-\nu) / 2} \bar{a}(t, s) \varphi^{\prime}(s)^{-(1-\nu) / 2} \\
=\varphi^{\prime}(t)^{(1-\nu) / 2} a(\varphi(t), \varphi(s)) \varphi^{\prime}(s)^{(1+\nu) / 2} \Phi(t, s)^{-\nu} \\
\widetilde{b}(t, s)=\varphi^{\prime}(t)^{(1-\nu) / 2} \bar{b}(t, s) \varphi^{\prime}(s)^{-(1-\nu) / 2}=\varphi^{\prime}(t)^{(1-\nu) / 2}[b(\varphi(t), \varphi(s)) \\
\left.+a(\varphi(t), \varphi(s)) \Phi(t, s)^{-\nu}\left(|t-s|^{-\nu}-\widetilde{g}(t-s)\right)\right] \varphi^{\prime}(s)^{(1+\nu) / 2} .
\end{gathered}
$$

Besides the equality $\widetilde{u}(t)=\varphi^{\prime}(t)^{(1-\nu) / 2} \bar{u}(t)$, the solutions of equations $(2.5)$ and (2.6) satisfy the integral relation

$$
\bar{u}(t)=\int_{0}^{1}\left(a_{\star}(t, s) \widetilde{g}(t-s)+b_{\star}(t, s)\right) \widetilde{u}(s) d s+\bar{f}(t), \quad 0 \leq t \leq 1,
$$

where

$$
\begin{gathered}
a_{\star}(t, s)=a(\varphi(t), \varphi(s)) \Phi(t, s)^{-\nu} \varphi^{\prime}(s)^{(1+\nu) / 2}, \quad b_{\star}(t, s)=[b(\varphi(t), \varphi(s)) \\
\left.+a(\varphi(t), \varphi(s)) \Phi(t, s)^{-\nu}\left(|t-s|^{-\nu}-\widetilde{g}(t-s)\right)\right] \varphi^{\prime}(s)^{(1+\nu) / 2} .
\end{gathered}
$$

The values of polynomials $\varphi(t)$ and $\Phi(t, s)$ at one point can be computed in $O(1)$ arithmetical operations, see [25] for corresponding procedures. Hence 
the values of $\bar{a}(t, s), \widetilde{a}(t, s), a_{\star}(t, s), \bar{b}(t, s), \widetilde{b}(t, s), b_{\star}(t, s)$ at one point can be computed at the cost of $O(1)$ arithmetical operations provided that the values $a(\varphi(t), \varphi(s))$ and $b(\varphi(t), \varphi(s))$ are given. Using a uniform grid for $t$ and $s$, $a(x, y)$ and $b(x, y)$ must be given or evaluated on a graded grid with respect to $x$ and $y$. Due to $(2.3),|t-s|^{-\nu}-\widetilde{g}(t-s)=0$ in the vicinity of the diagonal $t=s$, thus $\bar{b}(t, s), \widetilde{b}(t, s)$ and $b_{\star}(t, s)$ are regular on the diagonal $t=s$ of the square $0 \leq t, s \leq 1$ but there appear point singularities at $(0,1)$ and $(1,0)$ of the term $\widetilde{g}(t-s)$. Further, $\Phi(t, s)$ vanishes at $(0,0)$ and $(1,1)$ causing there point singularities of $\Phi(t, s)^{-\nu}$ of the order $(t+s)^{-(r-1) \nu}$ and $((1-t)+(1-s))^{-(r-1) \nu}$, respectively (see [25]):

$$
\left|\left(\frac{\partial}{\partial s}\right)^{j}(\Phi(t, s))^{-\nu}\right| \leq c(t+s)^{-\nu(r-1)-j}((1-t)+(1-s))^{-\nu(r-1)-j},
$$

where $j=0,1 \ldots, 2 m$; for $r>2$ these singularities are more strong than the singularities of $g^{(j)}$, see $(2.2)$. All these four point singularities can be suppressed by the factors $\varphi^{\prime}(t)^{(1-\nu) / 2}$ and $\varphi^{\prime}(s)^{(1+\nu) / 2}$ in the expressions for $\widetilde{a}, \widetilde{b}$ and by the factor $\varphi^{\prime}(s)^{(1+\nu) / 2}$ in the expressions for $a_{\star}, b_{\star}$. Actually, an analysis shows that the functions $\widetilde{a}, \tilde{b}, a_{\star}$ and $b_{\star}$ can be extended so that these extensions (which we denote again by $\left.\widetilde{a}, \widetilde{b}, a_{\star}, b_{\star}\right)$ belong to $C^{2 m}([0,1] \times[0,1])$ provided that the smoothing parameter $\mathrm{r}$ is sufficiently large: the condition

$$
r-1>4 m /(1-\nu)
$$

is sufficient. Namely, under condition (2.13) it holds that

$$
\left|\left(\frac{\partial}{\partial s}\right)^{j}\left(\Phi(t, s)^{-\nu}\right)\right|\left|\left(\frac{d}{d s}\right)^{2 m-j}\left(\varphi^{\prime}(s)^{(1+\nu) / 2}\right)\right| \rightarrow 0 \text { as } s \rightarrow 0,
$$

for any $t \in[0,1]$ and $j=0,1, \ldots, 2 m$.

Indeed, let $t \in[0,1], 0<s \leq 1 / 2$ and $j=0,1, \ldots, 2 m$. Then we obtain from (2.12) the estimate $\left|\left(\frac{\partial}{\partial s}\right)^{j}\left(\Phi(t, s)^{-\nu}\right)\right| \leq c s^{-\nu(r-1)-j}$, and from (2.4) the estimate

$$
\begin{aligned}
\left(\frac{d}{d s}\right)^{2 m-j}\left(\varphi^{\prime}(s)^{(1+\nu) / 2}\right) & =\left(\frac{d}{d s}\right)^{2 m-j}\left[\left(s^{r-1}(1-s)^{r-1}\right) / c_{*}\right]^{(1+\nu) / 2} \\
& \leq c_{1} s^{(r-1)((1+\nu) / 2)-(2 m-j)} .
\end{aligned}
$$

Therefore,

$$
\left|\left(\frac{\partial}{\partial s}\right)^{j}\left(\Phi(t, s)^{-\nu}\right) \|\left(\frac{d}{d s}\right)^{2 m-j}\left(\varphi^{\prime}(s)^{(1+\nu) / 2}\right)\right| \leq c_{2} s^{(r-1)((1-\nu) / 2)-2 m},
$$

with $(r-1)((1-\nu) / 2)-2 m>0$, if $r-1>4 m /(1-\nu)$, and (2.14) follows. Similarly, by symmetry,

$$
\left|\left(\frac{\partial}{\partial s}\right)^{j}\left(\Phi(t, s)^{-\nu}\right)\right|\left|\left(\frac{d}{d s}\right)^{2 m-j}\left(\varphi^{\prime}(s)^{(1+\nu) / 2)}\right)\right| \rightarrow 0 \text { as } s \rightarrow 1,
$$


for any $t \in[0,1]$ and $j=0,1, \ldots, 2 m$. Thus, the derivatives

$$
\left(\frac{\partial}{\partial s}\right)^{j}\left(\Phi(t, s)^{-\nu}\right)\left(\frac{d}{d s}\right)^{2 m-j}\left(\varphi^{\prime}(s)^{(1+\nu) / 2)}\right), \quad(j=0,1, \ldots, 2 m)
$$

have under the condition (2.13) no singularities at points $(t, s)=(0,0)$ and $(t, s)=(1,1)$ which, in turn yields that $\widetilde{a}, \widetilde{b}, a_{\star}, b_{\star} \in C^{2 m}([0,1] \times[0,1])$.

Moreover, under the condition (2.13),

$$
\left.\left(\frac{\partial}{\partial t}\right)^{j}\left(\frac{\partial}{\partial s}\right)^{k} \widetilde{a}(t, s)\right|_{(t, s) \in \Gamma}=0,\left.\quad\left(\frac{\partial}{\partial t}\right)^{j}\left(\frac{\partial}{\partial s}\right)^{k} \widetilde{b}(t, s)\right|_{(t, s) \in \Gamma}=0
$$

with $0 \leq j+k \leq 2 m$, and

$$
\begin{aligned}
& \left.\left(\frac{\partial}{\partial s}\right)^{k} a_{\star}(t, s)\right|_{s=0}=\left.\left(\frac{\partial}{\partial s}\right)^{k} a_{\star}(t, s)\right|_{s=1}=0, \\
& \left.\left(\frac{\partial}{\partial s}\right)^{k} b_{\star}(t, s)\right|_{s=0}=\left.\left(\frac{\partial}{\partial s}\right)^{k} b_{\star}(t, s)\right|_{s=1}=0, k=0,1, \ldots, 2 m,
\end{aligned}
$$

where $\Gamma$ is the boundary of the square $0 \leq t, s \leq 1$. Relations (2.15) allow to treat $\widetilde{a}$ and $\widetilde{b}$ as $C^{2 m}(\mathbb{R} \times \mathbb{R})$-smooth 1-biperiodic functions, i.e., $\widetilde{a}, \widetilde{b} \in \widetilde{C}^{2 m}(\mathbb{R} \times$ $\mathbb{R})$. According to $(2.16) a_{\star}(t, s)$ and $b_{\star}(t, s)$ can be treated as $C^{2 m}([0,1] \times \mathbb{R})$ smooth functions which are 1-periodic with respect to s. Further, (2.13) and $f \in C^{m, \nu}(0,1)$ imply that $\bar{f}, \tilde{f} \in C^{m}[0,1]$,

$$
\begin{aligned}
& \widetilde{f}^{(j)}(0)=\widetilde{f}^{(j)}(1)=0, \quad j=0, \ldots, m ; \quad \bar{f}^{(j)}(0)=\bar{f}^{(j)}(1)=0, \quad j=1, \ldots, m, \\
& \|\widetilde{f}\|_{C^{m}} \leq c\|\bar{f}\|_{C^{m}} \leq c^{\prime}\|f\|_{C^{m, \nu}},
\end{aligned}
$$

where the constants $c$ and $c^{\prime}$ are independent of $f$; in particular, we can treat $\widetilde{f}$ as a $C^{m}(\mathbb{R})$-smooth 1-periodic function, i.e., $\widetilde{f} \in \widetilde{C}^{m}(\mathbb{R})$.

\section{Preliminaries}

\subsection{Some notations}

We will use the following notations for spaces and norms in them: $C^{m}[0,1]$ is the space of $m$ times $(m \geq 0)$ continuously differentiable functions $u$ on $[0,1]$, $\|u\|_{C^{m}}=\sum_{k=0}^{m}\left\|u^{(k)}\right\|_{\infty}=\sum_{k=0}^{m} \max _{0 \leq x \leq 1}\left|u^{(k)}(x)\right| ; \widetilde{C}^{m}=\widetilde{C}^{m}(\mathbb{R})$ is the space of 1-periodic functions $\widetilde{u}$ on $\mathbb{R}$ with the same norm $\|\widetilde{u}\|_{C^{m}} ; C=C[0,1]=$ $C^{0}[0,1], \quad \widetilde{C}=\widetilde{C}(\mathbb{R})=\widetilde{C}^{0}(\mathbb{R}) ;$ the norm in $L^{p}(0,1), 1 \leq p \leq \infty$, is denoted

$$
\begin{aligned}
& \left.\|u\|_{p}=\left(\int_{0}^{1}|u(x)|^{p} d x\right)\right)^{1 / p}, \quad u \in L^{p}(0,1), \quad 1 \leq p<\infty, \\
& \|u\|_{\infty}=\operatorname{vraisup}_{0 \leq x \leq 1}|u(x)|, \quad u \in L^{\infty}(0,1) ;
\end{aligned}
$$

$\widetilde{L}^{p}=\widetilde{L}^{p}(\mathbb{R}), \quad p \in[1, \infty]$, is the space of 1-periodic functions $\widetilde{u}$ with the same norm: $\|\widetilde{u}\|_{p}^{p}=\int_{0}^{1}|\widetilde{u}(x)|^{p} d x, \quad p \in[1, \infty) ;\|\widetilde{u}\|_{\infty}=\operatorname{vraisup}_{0 \leq x \leq 1}|\widetilde{u}(x)| ;$ 
$W^{m, p}(0,1), 1 \leq p \leq \infty$, is the Sobolev space of functions $u$ on $(0,1)$ equipped with the norm

$$
\|u\|_{W^{m, p}}=\left(\sum_{k=0}^{m}\left\|u^{(k)}\right\|_{p}^{p}\right)^{1 / p}, \quad 1 \leq p<\infty ; \quad\|u\|_{W^{m, \infty}}=\sum_{k=0}^{m}\left\|u^{(k)}\right\|_{\infty}
$$

$\widetilde{W}^{m, p}=\widetilde{W}^{m, p}(\mathbb{R}), 1 \leq p \leq \infty$, is the Sobolev space of 1 -periodic functions $\widetilde{u}$ with the same norm $\|\widetilde{u}\|_{W^{m, p}} ; \mathcal{H}^{m, \lambda}=\mathcal{H}^{m, \lambda}[0,1], 0<\lambda \leq 1$, is the Hoelder space of functions $u \in C^{m}[0,1]$ with $u^{(m)}$ satisfying the Hoelder condition with the exponent $\lambda$;

$$
\|u\|_{\mathcal{H}^{m, \lambda}}=\|u\|_{C^{m}}+\sup \left\{\frac{\left|u^{(m)}(x)-u^{(m)}(y)\right|}{|x-y|^{\lambda}}: x, y \in[0,1], x \neq y\right\} ;
$$

$\widetilde{\mathcal{H}}^{m, \lambda}(\mathbb{R}), 0<\lambda \leq 1$, is the Hoelder space of 1 -periodic functions $\widetilde{u}$ with the same norm $\|\widetilde{u}\|_{\mathcal{H}^{m, \lambda}}$.

Next we introduce some known results about trigonometric interpolation and local interpolation by algebraic polynomials.

\subsection{Representation forms for a trigonometric polynomial}

For $n \in \mathbb{N}$, we denote

$$
\mathbb{Z}_{n}=\{k \in \mathbb{Z}:-n / 2<k \leq n / 2\}, \quad \mathcal{T}_{n}=\operatorname{span}\left\{e^{\mathrm{i} k 2 \pi t}: k \in \mathbb{Z}_{n}\right\} .
$$

Thus $\mathcal{T}_{n}$ consists of trigonometric polynomials, $\operatorname{dim} \mathcal{T}_{n}=n$. There are two possible representations of $v_{n} \in \mathcal{T}_{n}$ - through its Fourier coefficients $\hat{v}_{n}(k)$, $\hat{v}_{n}(k)=\int_{0}^{1} v_{n}(t) e^{-\mathrm{i} k 2 \pi t} d t, k \in \mathbb{Z}_{n}$, and through its nodal values $v_{n}\left(j n^{-1}\right)$, $j=0, \ldots, n-1$ :

$$
\begin{aligned}
& v_{n}(t)=\sum_{k \in \mathbb{Z}_{n}} \hat{v}_{n}(k) e^{\mathrm{i} k 2 \pi t} \\
& v_{n}(t)=\sum_{j=0}^{n-1} v_{n}\left(j n^{-1}\right) \phi_{n, j}(t), \quad \phi_{n, j}(t)=\frac{1}{n} \sum_{k \in \mathbb{Z}_{n}} e^{\mathrm{i} k 2 \pi\left(t-j n^{-1}\right)} .
\end{aligned}
$$

The functions $\phi_{n, j} \in \mathcal{T}_{n}, j=0,1, \ldots, n-1$, called fundamental trigonometric polynomials, satisfy

$$
\phi_{n, j}\left(l n^{-1}\right)=\delta_{j, l}, \quad j, l=0, \ldots, n-1,
$$

where $\delta_{j, l}$ is the Kronecker symbol. Having the nodal values of $v_{n} \in \mathcal{T}_{n}$ in hand, its Fourier coefficients are given by

$$
\hat{v}_{n}(k)=\int_{0}^{1} v_{n}(t) e^{-\mathrm{i} k 2 \pi t} d t=\frac{1}{n} \sum_{j=0}^{n-1} v_{n}\left(j n^{-1}\right) e^{-\mathrm{i} k 2 \pi j n^{-1}}, k \in \mathbb{Z}_{n}, \text { or } \hat{v}_{n}=\mathcal{F}_{n} \underline{v}_{n}
$$

where $\hat{v}_{n}$ is the vector with components $\hat{v}_{n}(k), k \in \mathbb{Z}_{n}, \underline{v}_{n}$ is the vector with components $v_{n}\left(j n^{-1}\right), j=0, \ldots, n-1$, and $\mathcal{F}_{n}$ is the discrete Fourier transform. 
Conversely, having the Fourier coefficients in hand, the nodal values of $v_{n} \in \mathcal{T}_{n}$ are given by the inverse discrete Fourier transform following from (3.1):

$$
v_{n}\left(j n^{-1}\right)=\sum_{k \in \mathbb{Z}_{n}} \hat{v}_{n}(k) e^{\mathrm{i} k 2 \pi j n^{-1}}, j=0, \ldots, n-1, \quad \text { or } \quad \underline{v}_{n}=\mathcal{F}_{n}^{-1} \hat{v}_{n} .
$$

So we can change the form of representation of a trigonometric polynomial where needed. In usual matrix calculus, an application of $\mathcal{F}_{n}$ or $\mathcal{F}_{n}^{-1}$ costs $n^{2}$ flops. Using fast Fourier transform (FFT) techniques, both transforms can be implemented in $\mathcal{O}(n \log n)$ flops. See [21] for further discussions.

\subsection{Trigonometric orthogonal and interpolation projections}

For $v \in L^{1}(0,1)$ and $n \in \mathbb{N}$ the Fourier projection $P_{n} v$ is defined by

$$
\left(P_{n} v\right)(t)=\sum_{k \in \mathbb{Z}_{n}} \hat{v}(k) e^{\mathrm{i} k 2 \pi t}, \quad \hat{v}(k)=\int_{0}^{1} v(t) e^{-\mathrm{i} k 2 \pi t} d t, k \in \mathbb{Z} .
$$

For $v \in L^{2}(0,1), P_{n} v$ is the orthogonal projection of $v$ onto $\mathcal{T}_{n}$. Clearly, we have $\left(P_{n} v\right)^{(m)}=P_{n} v^{(m)}$ for $v \in \widetilde{C}^{m}(\mathbb{R})$. For $v \in \widetilde{C}(\mathbb{R})$, the interpolation projection $Q_{n} v$ is defined by the requirements

$$
Q_{n} v \in \mathcal{T}_{n}, \quad\left(Q_{n} v\right)\left(j n^{-1}\right)=v\left(j n^{-1}\right), \quad j=0, \ldots, n-1 .
$$

Due to $(3.2),\left(Q_{n} v\right)(t)=\sum_{j=0}^{n-1} v\left(j n^{-1}\right) \phi_{n, j}(t)$, hence the Fourier coefficients $\left(\widehat{Q_{n} v}\right)(k), k \in \mathbb{Z}_{n}$, of $Q_{n} v$ can be computed from the sample values $v\left(j n^{-1}\right)$, $j=0, \ldots, n-1$, by FFT in $\mathcal{O}(n \log n)$ flops. It is known [35] that

$$
\begin{gathered}
\left\|P_{n}\right\|_{\widetilde{C}(\mathbb{R}) \rightarrow \widetilde{C}(\mathbb{R})} \leq c \log n, \quad\left\|P_{n}\right\|_{L^{p}(0,1) \rightarrow L^{p}(0,1)} \leq c_{p}, \quad 1<p<\infty \\
\left\|Q_{n}\right\|_{\widetilde{C}(\mathbb{R}) \rightarrow \widetilde{C}(\mathbb{R})} \leq c \log n, \quad\left\|Q_{n}\right\|_{\widetilde{C}(\mathbb{R}) \rightarrow L^{p}(0,1)} \leq c_{p}, \quad 1 \leq p<\infty
\end{gathered}
$$

Here the constants $c$ and $c_{p}$ are independent of $n$; we always assume that $n \geq 2$ in order to simplify the citing of estimates containing the factor $\log n$. The following estimates are direct consequences of (3.3) and (3.4):

$$
\begin{aligned}
& \left\|v-P_{n} v\right\|_{\infty} \leq c_{m} n^{-m}(\log n)\left\|v^{(m)}\right\|_{\infty}, \quad v \in \widetilde{C}^{m}(\mathbb{R}), m \in \mathbb{N}, \\
& \left\|v-P_{n} v\right\|_{p} \leq c_{m, p} n^{-m}\left\|v^{(m)}\right\|_{p}, \quad v \in \widetilde{W}^{m, p}(\mathbb{R}), m \in \mathbb{N}, 1<p<\infty, \\
& \left\|v-Q_{n} v\right\|_{\infty} \leq c_{m} n^{-m}(\log n)\left\|v^{(m)}\right\|_{\infty}, \quad v \in \widetilde{C}^{m}(\mathbb{R}), m \in \mathbb{N}, \\
& \left\|v-Q_{n} v\right\|_{\infty} \leq c_{m, \lambda} n^{-m-\lambda}(\log n)\left\|v^{(m)}\right\|_{\mathcal{H}^{m, \lambda}}, \quad v \in \widetilde{\mathcal{H}}^{m, \lambda}, m \geq 0, \\
& \left\|v-Q_{n} v\right\|_{p} \leq c_{m, p} n^{-m}\left\|v^{(m)}\right\|_{\infty}, \quad v \in \widetilde{C}^{m}(\mathbb{R}), \quad m \in \mathbb{N}, 1 \leq p<\infty .
\end{aligned}
$$

The constants in the estimates (3.5)-(3.7) are independent of $n$ and $v$.

\subsection{Local interpolation by polynomials}

For $n \in \mathbb{N}$ denote $h=1 / n$. To $u \in \widetilde{C}(\mathbb{R})$, assign the piecewise polynomial interpolant $\Pi_{h, m} u \in \widetilde{C}(\mathbb{R})$ which is defined on every subinterval $[i h,(i+1) h]$ 
independently of other subintervals as an algebraic polynomial $u_{i}$ of degree $m-1$ (of order $\mathrm{m}$ ) such that $u_{i}(j h)=u(j h)$ for $j-i \in \mathbb{Z}_{m}, m \in \mathbb{N}$. Well known error estimates for a polynomial interpolant (cf. [1,10]) yield the following results.

Lemma 1. (i) For $u \in \widetilde{C}^{m}(\mathbb{R})(m \in \mathbb{N})$, it holds

$$
\begin{aligned}
& \left\|u-\Pi_{h, m} u\right\|_{\infty} \leq c n^{-m}\left\|u^{(m)}\right\|_{\infty}, \\
& \max _{x \in \mathbb{R}}\left|u^{(k)}(x)-\left(\Pi_{h, m} u\right)^{(k)}(x)\right| \leq c n^{-(m-k)}\left\|u^{(m)}\right\|_{\infty}, \quad k=0, \ldots, m-1 ;
\end{aligned}
$$

at $x=i h, \quad i \in \mathbb{Z}$, this holds for both one side limits of $\left(\Pi_{h, m} u\right)^{(k)}(x)$.

(ii) For $u \in \widetilde{\mathcal{H}}^{m, \lambda}(\mathbb{R}), m \in\{0\} \cup \mathbb{N}, 0<\lambda<1$, it holds

$$
\left\|u-\Pi_{h, m+1} u\right\|_{\infty} \leq c n^{-m-\lambda}\left\|u^{(m)}\right\|_{\mathcal{H}^{m, \lambda}} .
$$

\section{An approximate method for the periodized problem}

In Section 2 we obtained an 1-periodic problem (2.6) with $m \in \mathbb{N}, \widetilde{f} \in \widetilde{C}^{m}(\mathbb{R})$, $\widetilde{a}, \widetilde{b} \in \widetilde{C}^{2 m}(\mathbb{R} \times \mathbb{R})$, and $\widetilde{g} \in \widetilde{C}^{2 m}(\mathbb{R} \backslash \mathbb{Z})$ satisfying (2.2) and (2.3). Fast solving of such a problem has been examined in [14]. In this section we recall and modify some results of [14] applied to equation (2.6).

For $n \in \mathbb{N}$, let $n^{\prime} \in \mathbb{N}$ be such that

$$
n^{\prime} \geq 2 n, \quad n^{\prime} \sim n^{\tau}, 2 m /(m+1-\nu)<\tau<2,
$$

where $n^{\prime} \sim n^{\tau}$ means that $n^{\prime} / n^{\tau} \rightarrow 1$ as $n \rightarrow \infty$. We approximate equation (2.7) (equation (2.6)) by the equation

$$
\widetilde{u}_{n, n^{\prime}}=\widetilde{T}_{n, n^{\prime}} \widetilde{u}_{n, n^{\prime}}+P_{n^{\prime}} \tilde{f},
$$

where $P_{n^{\prime}} \tilde{f}$ is an approximation for the free term $\tilde{f}$ in equation (2.7) and

$$
\widetilde{T}_{n, n^{\prime}}=Q_{2 n}\left(\widetilde{A}_{2 n}+\widetilde{B}_{2 n}\right) P_{n}+Q_{n^{\prime}} \widetilde{A}_{n}^{(m)}\left(I-P_{n}\right)
$$

is an approximation for the integral operator $\widetilde{T}=\widetilde{A}+\widetilde{B}$ of equation (2.6) (see (2.8) and (2.9)). Here $P_{n}$ and $Q_{n}$ are respectively the orthogonal and interpolation projection operators introduced in Section 3.3,

$$
\begin{aligned}
& \left(\widetilde{A}_{n} v\right)(t)=\int_{0}^{1} \widetilde{g}(t-s) Q_{n ; s}(\widetilde{a}(t, s) v(s)) d s \\
& \left(\widetilde{B}_{n} v\right)(t)=\int_{0}^{1} Q_{n ; s}(\widetilde{b}(t, s) v(s)) d s
\end{aligned}
$$

( $s$ in the index of $Q_{n ; s}$ refers that $Q_{n}$ is applied w.r.t. the argument $s$ ),

$$
\begin{aligned}
& \widetilde{A}_{n}^{(m)}=\sum_{j=0}^{m-1} M_{\alpha_{j}, n} G_{j}, \quad\left(G_{j} v\right)(t)=\int_{0}^{1} \widetilde{g}_{j}(t-s) v(s) d s, \widetilde{g}_{j}(t)=\left(e^{-\mathrm{i} 2 \pi t}-1\right)^{j} \widetilde{g}(t), \\
& \left(M_{\alpha_{j}, n} v\right)(t)=\alpha_{j, n}(t) v(t), \quad \alpha_{j, n}(i / n)=\left.\frac{1}{j !} L_{j, n} \widetilde{a}(i / n, s)\right|_{s=i / n},
\end{aligned}
$$


where $i=0, \ldots, n$ and $L_{j, n}(j \geq 1)$ is some difference approximation on the grid $i / n(i \in \mathbb{Z})$ of the differential operator $L_{j}=\prod_{l=0}^{j-1}\left((2 \pi \mathrm{i})^{-1} \frac{\partial}{\partial s}-l I\right)$ of the accuracy $\left|\left(L_{j} v\right)(s)-\left(L_{j, n} v\right)(s)\right| \leq c n^{-2 m+j}\left\|v^{(2 m)}\right\|_{\infty}, s \in \mathbb{R}$; for $j=0$ we put $L_{0}=L_{0, n}=I$. The matrix form of $\widetilde{A}_{n}^{(m)}$ needs also the values $\alpha_{j, n}\left(j / n^{\prime}\right)$, $j=0, \ldots, n^{\prime}-1$, which we approximate via interpolation of $\alpha_{j, n}(i / n), i=$ $0, \ldots, n$, by splines of degree $2 m$. See [14] for more detailed comments on the listed operators.

Lemma 2. Assume (A2) (which implies that $\widetilde{a}, \widetilde{b} \in \widetilde{C}^{2 m}(\mathbb{R} \times \mathbb{R})$ ). Let $n, n^{\prime} \in$ $\mathbb{N}$, and let $n^{\prime} \sim n^{\tau}, \tau>1$. Then for $1 /(1-\nu)<p<\infty$,

$$
\left\|\widetilde{T}\left(I-P_{n^{\prime}}\right)\right\|_{\widetilde{L}^{p} \rightarrow \widetilde{C}} \leq c_{p} n^{-\tau(1-\nu-1 / p)} ;
$$

for $1 /(1-\nu)<p \leq \infty, 0<\lambda<1-\nu-p^{-1}$,

$$
\left\|\widetilde{T}-\widetilde{T}_{n, n^{\prime}}\right\|_{\widetilde{L}^{p} \rightarrow \widetilde{C}} \leq c_{p, \lambda}\left(n^{-m}+n^{-\tau \lambda}\right) \log n
$$

for $1 /(1-\nu)<p<\infty, 0<\lambda<1-\nu-p^{-1}, v \in \widetilde{W}^{m, p}(\mathbb{R})$,

$$
\begin{aligned}
& \left\|\widetilde{T} v-\widetilde{T}_{n, n^{\prime}} v\right\|_{p} \leq c_{p, \lambda}\left(n^{-2 m}+n^{-\tau(m+\lambda)}(\log n)\right)\|v\|_{W^{m, p}}, \\
& \left\|\widetilde{T} v-\widetilde{T}_{n, n^{\prime}} v\right\|_{\infty} \leq c_{p, \lambda}\left(n^{-2 m}+n^{-\tau(m+\lambda)}\right)(\log n)\|v\|_{W^{m, p}},
\end{aligned}
$$

and, strengthening (A2) to the assumption (A2'),

$$
\left\|\widetilde{T} v-\widetilde{T}_{n, n^{\prime}} v\right\|_{\infty} \leq c_{p, \lambda}\left(n^{-2 m-\min \{\mu, 1-\nu-1 / p\}}+n^{-\tau(m+\lambda)}\right)(\log n)\|v\|_{W^{m, p}} .
$$

Proof. Let $1 /(1-\nu)<p<\infty$. Since $I-P_{n}$ as an orthogonal projector in $L^{2}(0,1)$ is self-adjoint, it holds for $t \in[0,1]$ that

$$
\begin{gathered}
\left(\widetilde{A}\left(I-P_{n}\right) v\right)(t)=\int_{0}^{1} \widetilde{a}(t, s) \widetilde{g}(t-s)\left(\left(I-P_{n}\right) v\right)(s) d s \\
=\int_{0}^{1}\left(I-P_{n ; s}\right)(\widetilde{a}(t, s) \widetilde{g}(t-s)) v(s) d s \\
\left|\left(\widetilde{A}\left(I-P_{n}\right) v\right)(t)\right| \leq\left\|\left(I-P_{n}\right)(\widetilde{a}(t, \cdot) \widetilde{g}(t-\cdot))\right\|_{q}\|v\|_{p}, \quad p^{-1}+q^{-1}=1 .
\end{gathered}
$$

Using (2.2) for $j=0$ and $j=1$, it is easy to check that for $|\sigma| \leq \delta$

$$
\left(\int_{0}^{1}|\widetilde{a}(t, s+\sigma) \widetilde{g}(t-s-\sigma)-\widetilde{a}(t, s) \widetilde{g}(t-s)|^{q} d s\right)^{1 / q} \leq c|\sigma|^{1-\nu-1 / p} .
$$

By Nikolski's inequality (formulated in [14] as Lemma 2.2), estimate (4.9) implies that

$$
\begin{gathered}
\left\|\left(I-P_{n}\right)(\widetilde{a}(t, \cdot) \widetilde{g}(t-\cdot))\right\|_{q} \leq c n^{-(1-\nu-1 / p)}, \quad t \in[0,1], \\
\left\|\widetilde{T}\left(I-P_{n}\right)\right\|_{\widetilde{L}^{p} \rightarrow \widetilde{C}} \leq c_{p} n^{-(1-\nu-1 / p)},
\end{gathered}
$$

which for $n^{\prime} \sim n^{\tau}$ yields (4.4). Estimates (4.5)-(4.8) are established in Lemma 3.5 of [14]; in the estimate (4.5) we corrected a misprint of [14]. 
Theorem 1. Assume (A1)-(A3). Moreover, assume that the smoothing parameter $r$ in (2.4) satisfies (2.13) and the dimension parameter $n^{\prime}$ satisfies (4.1). Then equations (1.1) and (2.6) have unique solutions $u \in C^{m, \nu}(0,1)$ and $\widetilde{u} \in \widetilde{C}^{m}(\mathbb{R})$, respectively. These solutions are connected by the relation $\widetilde{u}(t)=\varphi^{\prime}(t)^{(1-\nu) / 2} u(\varphi(t)) ;$ for sufficiently large $n$, say $n \geq n_{0}$, equation (4.2) has a unique solution $\widetilde{u}_{n, n^{\prime}} \in \mathcal{T}_{n^{\prime}}$, and

$$
\begin{aligned}
\left\|\widetilde{u}-\widetilde{u}_{n, n^{\prime}}\right\|_{\infty} & \leq c n^{-\tau m}(\log n)\|\widetilde{f}\|_{C^{m}}, \\
\left\|\widetilde{u}-\widetilde{u}_{n, n^{\prime}}\right\|_{p} & \leq c^{\prime} n^{-\tau m}\|\widetilde{f}\|_{W^{m, p}}, \quad 1 /(1-\nu)<p<\infty
\end{aligned}
$$

with some positive constants $c$ and $c^{\prime}$ which are independent of $n$ and $\tilde{f}$.

Proof. For $1 /(1-\nu)<p \leq \infty$, the operator $\widetilde{T}: \widetilde{L}^{p} \rightarrow \widetilde{C}(\mathbb{R})$ is compact, and since by the assumption (A3), the nullspace of $I-T$ is trivial in $C[0,1]$ implying that the null space of $I-\widetilde{T}$ is trivial in $\widetilde{C}(\mathbb{R})$, the bounded inverse $(I-\widetilde{T})^{-1}: \widetilde{L}^{p} \rightarrow \widetilde{L}^{p}$ exists. Hence equation (2.6) and with it also (1.1) are uniquely solvable. Further, due to (4.5), $\left\|\widetilde{T}-\widetilde{T}_{n, n^{\prime}}\right\|_{\widetilde{L}^{p} \rightarrow \widetilde{L}^{p}} \rightarrow 0$ as $n \rightarrow \infty$ for $1 /(1-\nu)<p \leq \infty$, hence for sufficiently large $n$, say $n \geq n_{0}$, also $\left(I-\widetilde{T}_{n, n^{\prime}}\right)^{-1}$ : $\widetilde{L}^{p} \rightarrow \widetilde{L}^{p}$ exists and is uniformly bounded in $n$ :

$$
\left\|\left(I-\widetilde{T}_{n, n^{\prime}}\right)^{-1}\right\|_{\widetilde{L}^{p} \rightarrow \widetilde{L}^{p}} \leq c_{p}, n \geq n_{0}, 1 /(1-\nu)<p \leq \infty .
$$

Thus equation (4.2) is uniquely solvable for $n \geq n_{0}$.

Clearly, $\left(I-\widetilde{T}_{n, n^{\prime}}\right)\left(\widetilde{u}-\widetilde{u}_{n, n^{\prime}}\right)=\widetilde{f}-P_{n^{\prime}} \widetilde{f}+\left(\widetilde{T}-\widetilde{T}_{n, n^{\prime}}\right) \widetilde{u}$, or

$$
\left.\widetilde{u}-\widetilde{u}_{n, n^{\prime}}=\left(I-\widetilde{T}_{n, n^{\prime}}\right)^{-1}\left(\widetilde{f}-P_{n^{\prime}} \widetilde{f}+\left(\widetilde{T}-\widetilde{T}_{n, n^{\prime}}\right) \widetilde{u}\right)\right), \quad n \geq n_{0} .
$$

Due to $(4.12),\left\|\widetilde{u}-\widetilde{u}_{n, n^{\prime}}\right\|_{p} \leq c_{p}\left(\left\|\widetilde{f}-P_{n^{\prime}} \widetilde{f}\right\|_{p}+\left\|\left(\widetilde{T}-\widetilde{T}_{n, n^{\prime}}\right) \widetilde{u}\right\|_{p}\right)$ with $n \geq$ $n_{0}, 1 /(1-\nu)<p \leq \infty$. Since $\widetilde{f} \in C^{m}(\mathbb{R})$, it follows from (3.5) and (3.6) that

$$
\begin{aligned}
& \left\|\widetilde{f}-P_{n} \widetilde{f}\right\|_{\infty} \leq c n^{-m}(\log n)\left\|\widetilde{f}^{(m)}\right\|_{\infty}, \\
& \left\|\tilde{f}-P_{n} \tilde{f}\right\|_{p} \leq c_{p} n^{-m}\left\|\tilde{f}^{(m)}\right\|_{p}, 1<p<\infty .
\end{aligned}
$$

This in view of (4.1) yields

$$
\left\|\widetilde{f}-P_{n^{\prime}} \widetilde{f}\right\|_{\infty} \leq c n^{-\tau m}(\log n)\|\widetilde{f}\|_{C^{m}},\left\|\widetilde{f}-P_{n^{\prime}} \widetilde{f}\right\|_{p} \leq c_{p} n^{-\tau m}\|\widetilde{f}\|_{W^{m, p}} .
$$

Applying also (4.7) and (4.6) with any $\lambda \in\left(0,1-\nu-p^{-1}\right)$ we arrive at estimates (4.10) and (4.11).

Following ideas of [14], the matrix form of equation (4.2) reads as

$$
\underline{u}_{n^{\prime}}=\underline{T}_{n^{\prime}} \underline{u}_{n^{\prime}}+\mathcal{F}_{n^{\prime}}^{-1} \widehat{\widetilde{\widetilde{f}}}_{n^{\prime}}
$$

where $\underline{u}_{n^{\prime}}=\left(\widetilde{u}_{n, n^{\prime}}(0), \widetilde{u}_{n, n^{\prime}}\left(1 / n^{\prime}\right), \ldots, \widetilde{u}_{n, n^{\prime}}\left(\left(n^{\prime}-1\right) / n^{\prime}\right)^{\text {tr }}\right.$ is the vector of values of $\widetilde{u}_{n, n^{\prime}}, \widehat{\widetilde{f}}_{n^{\prime}}$ is the vector of the Fourier coefficients $\widehat{\widetilde{f}}(k)=\int_{0}^{1} \widetilde{f}(t) e^{-\mathrm{i} k 2 \pi t} d t$ 
$\left(k \in \mathbb{Z}_{n^{\prime}}\right)$ of the function $\tilde{f}$, and

$$
\underline{T}_{n^{\prime}}=\mathcal{F}_{n^{\prime}}^{-1} \mathcal{E}_{n^{\prime}, 2 n} \mathcal{F}_{2 n}\left(\mathcal{A}_{2 n}+\mathcal{B}_{2 n}\right) \mathcal{F}_{2 n}^{-1} \mathcal{E}_{2 n, n} \mathcal{P}_{n, n^{\prime}} \mathcal{F}_{n^{\prime}}+\sum_{j=0}^{m-1} \mathcal{M}_{n^{\prime}, j} \mathcal{F}_{n^{\prime}}^{-1} \mathcal{G}_{n^{\prime}, j} \mathcal{F}_{n^{\prime}}
$$

is an $n^{\prime} \times n^{\prime}$ matrix. Here $\mathcal{F}_{n^{\prime}}, \mathcal{F}_{n^{\prime}}^{-1}$ and $\mathcal{F}_{2 n}, \mathcal{F}_{2 n}^{-1}$ are the Fourier transform matrices of dimensions $n^{\prime} \times n^{\prime}$ and $2 n \times 2 n$, respectively, changing the representation form of trigonometric polynomials (see Section 3 ); the projection-convolution $G_{j}\left(I-P_{n}\right): \mathcal{T}_{n^{\prime}} \rightarrow \mathcal{T}_{n^{\prime}} \quad(j=0, \ldots, m-1)$ is realized by the diagonal $n^{\prime} \times n^{\prime}$ matrix $\mathcal{G}_{n^{\prime}, j}$ with the diagonal elements

$$
\mathcal{G}_{n^{\prime}, j}(k, k)=0 \quad \text { for } k \in \mathbb{Z}_{n}, \quad \mathcal{G}_{n^{\prime}, j}(k, k)=\widehat{\widetilde{g}}_{j}(k) \quad \text { for } k \in \mathbb{Z}_{n^{\prime}} \backslash \mathbb{Z}_{n}
$$

the multiplication operator $M_{\alpha_{j}, n}(j=0, \ldots, m-1)$ is realized by the diagonal $n^{\prime} \times n^{\prime}$ matrix $\mathcal{M}_{n^{\prime}, j}$ with the diagonal elements

$$
\mathcal{M}_{n^{\prime}, j}(k, k)=\alpha_{j, n}\left(k / n^{\prime}\right), \quad k=0,1, \ldots, n^{\prime}-1 ;
$$

the projection $P_{n}: \mathcal{T}_{n^{\prime}} \rightarrow \mathcal{T}_{n}$ is realized by $n \times n^{\prime}$ matrix $\mathcal{P}_{n, n^{\prime}}$ consisting of the $n \times n$ identity matrix completed from left and right by $n \times \frac{n^{\prime}-n}{2}$ zero matrix; the embedding $\mathcal{T}_{n} \subset \mathcal{T}_{2 n}$ is realized by the $2 n \times n$ matrix $\mathcal{E}_{2 n, n}$ consisting of the $n \times n$ identity matrix complemented by $\frac{n}{2} \times n$ zero matrix from above and below (for simplicity we assume that $n$ and $n^{\prime}$ are even); the embedding $\mathcal{T}_{2 n} \subset \mathcal{T}_{n^{\prime}}$ is realized by the $n^{\prime} \times 2 n$ matrix $\mathcal{E}_{n^{\prime}, 2 n}$ consisting of the $2 n \times 2 n$ identity matrix completed by $n^{\prime} \times \frac{n^{\prime}-2 n}{2}$ zero matrix from above and below; finally, $\mathcal{A}_{2 n}=\left(a_{j, j^{\prime}}\right)$ and $\mathcal{B}_{2 n}=\left(b_{j, j^{\prime}}\right)$ are $2 n \times 2 n$ matrices with entries defined by the formulas

$$
\begin{aligned}
& a_{j, j^{\prime}}=\frac{1}{2 n} \widetilde{a}\left(\frac{j}{2 n}, \frac{j^{\prime}}{2 n}\right) \sigma_{j-j^{\prime}}, b_{j, j^{\prime}}=\frac{1}{2 n} \widetilde{b}\left(\frac{j}{2 n}, \frac{j^{\prime}}{2 n}\right), j, j^{\prime}=0, \ldots, 2 n-1, \\
& \sigma_{l}=\sum_{k \in \mathbb{Z}_{2 n}} e^{i k 2 \pi l /(2 n)} \widehat{\widetilde{g}}(k), \quad l=-2 n+1, \ldots, 2 n-1 .
\end{aligned}
$$

In (4.15) and (4.16) only the $2 n \times 2 n$ matrix $\mathcal{A}_{2 n}+\mathcal{B}_{2 n}$ and the diagonal $n^{\prime} \times n^{\prime}$ matrices $\mathcal{M}_{n^{\prime}, j}$ and $\mathcal{G}_{n^{\prime}, j}, j=0, \ldots, m-1$, and vector $\widehat{\widetilde{f}}_{n^{\prime}}$ depend on the data of problem (1.1), namely on $O\left(n^{2}\right)$ values $\widetilde{a}\left(\frac{j}{2 n}, \frac{j^{\prime}}{2 n}\right), \widetilde{b}\left(\frac{j}{2 n}, \frac{j^{\prime}}{2 n}\right)$, $j, j^{\prime}=0, \ldots, 2 n-1$, and on the Fourier coefficients $\widehat{\widetilde{g}}(k)\left(k \in \mathbb{Z}_{n^{\prime}+m-1}\right)$ of the function $\widetilde{g} \in \widetilde{C}^{2 m}(\mathbb{R} \backslash \mathbb{Z})$ defined due to $(2.1)$, which can be computed in $O\left(n^{2}\right)$ arithmetical operations with a very high accuracy $O\left(n^{-4 m}\right)$ from the values on a suitable graded grid consisting of $n^{2}$ points; see [14] for details. The application of $\mathcal{A}_{2 n}+\mathcal{B}_{2 n}$ to an $2 n$-vector costs $O\left(n^{2}\right)$, the application of FFT transformation matrices $\mathcal{F}_{n^{\prime}}$ and $\mathcal{F}_{n^{\prime}}^{-1}$ of dimension $n^{\prime} \times n^{\prime}$ to an $n^{\prime}$-vector $\operatorname{costs} O\left(n^{\prime} \log n\right)$, and the application of other matrices in $\underline{T}_{n^{\prime}}$ is cheaper. Thus the application of $\underline{T}_{n^{\prime}}$ to a $n^{\prime}$-vector costs $O\left(n^{2}\right)+O\left(n^{\prime} \log n\right)$ arithmetical operations. This enables to solve system (4.15) in $O\left(n^{2}\right)$ arithmetical operations by the two grid iteration method combined with the GMRES $[9,21,24,27]$ on the coarse level. 
It remains to comment on the computation of the Fourier coefficients $\widehat{\widetilde{f}}(k)$, $k \in \mathbb{Z}_{n^{\prime}}$. In [14], a more complicated approximation of the free term is used that restricts the computation of Fourier coefficients to $k \in \mathbb{Z}_{n}$; this approximation is not suitable for goals of the present paper. Nevertheless, the idea of [14] can be used to compute also the Fourier coefficients $\widehat{\widetilde{f}}(k), k \in \mathbb{Z}_{n^{\prime}}$ : in a special way described below, we approximate $\tilde{f}$ by a function $\tilde{f}_{n, n^{\prime \prime}}$ depending on the parameters $n$ and $n^{\prime \prime} \sim n^{\sigma}, \tau<\sigma<2$ (with $\tau$ from (4.1)) so that the Fourier coefficients $\widehat{\widetilde{f}}_{n, n^{\prime \prime}}$ of $\widetilde{f}_{n, n^{\prime \prime}}$ are for $k \in \mathbb{Z}_{n^{\prime}}$ (exactly) computable in $O\left(n^{2}\right)$ arithmetical operations and, with a $p \in(1 /(1-\nu), \infty)$,

$$
\left\|P_{n^{\prime}}\left(\tilde{f}-\tilde{f}_{n, n^{\prime \prime}}\right)\right\|_{p} \leq c n^{-2 m}\|\widetilde{f}\|_{C^{m}} .
$$

The following remark reveals that this accuracy occurs to be sufficient for our purposes.

Remark 3. Let $\widetilde{u}_{n, n^{\prime}}$ be the solution of equation (4.2), and let $\widetilde{u}_{n, n^{\prime}, n^{\prime \prime}}$ be the solution of the perturbed equation $\widetilde{u}_{n, n^{\prime}, n^{\prime \prime}}=\widetilde{T}_{n, n^{\prime}} \widetilde{u}_{n, n^{\prime}, n^{\prime \prime}}+P_{n^{\prime}} \widetilde{f}_{n, n^{\prime \prime}}$, where $\tilde{f}_{n, n^{\prime \prime}}$ satisfies $(4.17)$ with a $p \in(1 /(1-\nu), \infty)$. Then, for sufficiently large $n$,

$$
\left\|\widetilde{u}_{n, n^{\prime}}-\widetilde{u}_{n, n^{\prime}, n^{\prime \prime}}\right\|_{p} \leq c n^{-2 m}\|\widetilde{f}\|_{C^{m}} .
$$

This immediately follows from $\widetilde{u}_{n, n^{\prime}}-\widetilde{u}_{n, n^{\prime}, n^{\prime \prime}}=\left(I-\widetilde{T}_{n, n^{\prime}}\right)^{-1} P_{n^{\prime}}\left(\tilde{f}-\widetilde{f}_{n, n^{\prime \prime}}\right)$ and (4.12).

Let us describe how a suitable $\widetilde{f}_{n, n^{\prime \prime}}$ can be constructed. Using (smooth) splines of degree $m$ with knots $i / n^{2}, i \in \mathbb{Z}$, we construct in $O\left(n^{2}\right)$ arithmetical operations the interpolant or quasi-interpolant $\tilde{f}_{n}$ of $\tilde{f}$ which satisfies (cf. [25]) with a $p \in(1 /(1-\nu), \infty)$ the following inequalities:

$$
\left\|P_{n^{\prime}}\left(\tilde{f}-\tilde{f}_{n}\right)\right\|_{p} \leq c\left\|\tilde{f}-\tilde{f}_{n}\right\|_{\infty} \leq c^{\prime} n^{-2 m}\left\|\tilde{f}^{(m)}\right\|_{\infty},\left\|\widetilde{f}_{n}^{(m)}\right\|_{\infty} \leq c\|\widetilde{f}\|_{C^{m}} .
$$

Take a number $n^{\prime \prime} \sim n^{\sigma}, \tau<\sigma<2$ with $\tau$ from condition (4.1). Starting from $\widetilde{f}_{n}^{(0)}=\widetilde{f}_{n}$, compute the 1-periodic antiderivatives

$$
\widetilde{f}_{n}^{(-j)}(t)=\int_{0}^{t}\left(\widetilde{f}_{n}^{(-j+1)}(s)-\int_{0}^{1} \widetilde{f}_{n}^{(-j+1)}(s) d s\right) d s, \quad j=1, \ldots, l,
$$

where $l \geq \frac{2 m+\frac{1}{2} \tau}{\sigma-\tau}$, and put $\widetilde{f}_{n, n^{\prime \prime}}=\int_{0}^{1} \widetilde{f}_{n}(z) d z+\left(Q_{n^{\prime \prime}} \widetilde{f}_{n}^{(-l)}\right)^{(l)}$. The values $\widetilde{f}_{n}^{(-l)}\left(i / n^{\prime \prime}\right), i=0, \ldots, n^{\prime \prime}-1$, determining the interpolant $Q_{n^{\prime \prime}} \widetilde{f}_{n}^{(-l)}$ are available at the cost of $O\left(n^{2}\right)$ arithmetical operations. By FFT we can compute the Fourier coefficients $\left(\widehat{Q_{n^{\prime \prime}} \widetilde{f}_{n}^{(-}}\right)(k), k \in \mathbb{Z}_{n^{\prime \prime}}$, at the cost of $O\left(n^{\prime \prime} \log n\right)$ arithmetical operations. The Fourier coefficients of $P_{n^{\prime}} \widetilde{f}_{n, n^{\prime \prime}}$ can be picked from the equality

$$
\left(P_{n^{\prime}} \widetilde{f}_{n, n^{\prime \prime}}\right)(x)=\int_{0}^{1} \widetilde{f}_{n}(z) d z+\sum_{0 \neq k \in \mathbb{Z}_{n^{\prime}}}\left(\widehat{Q_{n^{\prime \prime}} \widetilde{f}_{n}^{(-l)}}\right)(k)(\mathrm{i} k 2 \pi)^{l} e^{\mathrm{i} k 2 \pi x}
$$


Repeating the argument of [14] we get the estimate

$$
\left\|P_{n^{\prime}}\left(\tilde{f}_{n}-\tilde{f}_{n, n^{\prime \prime}}\right)\right\|_{\infty} \leq \pi^{l}\left(n^{\prime}\right)^{l+\frac{1}{2}}\left\|\widetilde{f}_{n}^{(-l)}-Q_{n^{\prime \prime}} \tilde{f}_{n}^{(-l)}\right\|_{2} .
$$

Since $\left\|\widetilde{f}_{n}^{(-l)}-Q_{n^{\prime \prime}} \widetilde{f}_{n}^{(-l)}\right\|_{2} \leq c\left(n^{\prime \prime}\right)^{-(m+l)}\left\|\widetilde{f}_{n}\right\|_{W^{m, 2}} \leq c^{\prime} n^{-2 m}\|\widetilde{f}\|_{C^{m}}$, the estimate (4.17) follows:

$$
\left\|P_{n^{\prime}}\left(\tilde{f}-\widetilde{f}_{n, n^{\prime \prime}}\right)\right\|_{p} \leq\left\|P_{n^{\prime}}\left(\tilde{f}-\widetilde{f}_{n}\right)\right\|_{p}+\left\|P_{n^{\prime}}\left(\tilde{f}_{n}-\widetilde{f}_{n, n^{\prime \prime}}\right)\right\|_{p} \leq c n^{-2 m}\|\widetilde{f}\|_{C^{m}} .
$$

Our next step is to compute the approximate solution of equation (2.5) using integral relation (2.10) in which we replace $\widetilde{u}$, the solution of (2.6), by $\widetilde{u}_{n, n^{\prime}}$, the solution of (4.2). Recall that the kernel of integral operator in (2.10) is periodic only w.r.t. argument $s$, so we cannot immediately use the techniques of the present section. To make this possible, we first decompose the kernel extracting from it a periodic part and a polynomial part. This is done in Section 6 after some preliminaries (Section 5).

\section{Extracting a periodic and polynomial parts of a function}

Denote by $\mathbb{P}_{i}$ the space of polynomials $z$ of degree not exceeding $i+1$ and satisfying $\int_{0}^{1} z(t) d t=0$. There exist unique polynomials $z_{i} \in \mathbb{P}_{i}, i=0,1, \ldots$, such that

$$
z_{i}^{(k)}(1)-z_{i}^{(k)}(0)=\delta_{i, k} \text { (Kronecker symbol) }, \quad k=0,1,2, \ldots
$$

Indeed, fixing $i,(5.1)$ is trivially fulfilled for $k \geq i+1$, so we have to determine the coefficients $c_{i j}, j=0, \ldots, i+1$, of $z_{i}(t)=\sum_{j=0}^{i+1} c_{i j} t^{j}$ so that (5.1) holds for $k=0, \ldots, i$ and $\int_{0}^{1} z_{i}(t) d t=0$. These conditions yield an $i+2$ dimensional triangular system with nonzeroes on the main diagonal uniquely determining $c_{i j}, j=0, \ldots, i+1$. Clearly, $z_{0}(t)=t-\frac{1}{2}$. Having $z_{i}$ in hand and knowing that $z_{i+1}$ exists and is unique, it is easy to check that it satisfies the recursion

$$
z_{i+1}(t)=\int_{0}^{t} z_{i}(s) d s+\int_{0}^{1} s z_{i}(s) d s, \quad i=0,1, \ldots
$$

The first three polynomials $z_{i}$ are as follows:

$$
z_{0}(t)=t-\frac{1}{2}, \quad z_{1}(t)=\frac{1}{2} t^{2}-\frac{1}{2} t+\frac{1}{12}, \quad z_{2}(t)=\frac{1}{6} t^{3}-\frac{1}{4} t^{2}+\frac{1}{12} t .
$$

Denote by $\widetilde{C}^{m}[0,1]$ the subspace of functions $\widetilde{w} \in C^{m}[0,1]$ satisfying the periodic boundary conditions $\widetilde{w}^{(i)}(1)-\widetilde{w}^{(i)}(0)=0, i=0, \ldots, m$. Every function $w \in C^{m}[0,1]$ has the representation

$$
w=\widetilde{w}+w_{m}, w_{m}=\sum_{i=0}^{m}\left[w^{(i)}(1)-w^{(i)}(0)\right] z_{i} \in \mathbb{P}_{m}, \widetilde{w}=w-w_{m} \in \widetilde{C}^{m}[0,1] .
$$

Since an 1-periodic extension of a function $\widetilde{w} \in \widetilde{C}^{m}[0,1]$ is $C^{m}(\mathbb{R})$-smooth, we can identify $\widetilde{C}^{m}[0,1]$ with $\widetilde{C}^{m}(\mathbb{R})$. 


\section{An approximate method for equation (1.1) (equation $(2.5))$}

Let us apply decomposition (5.2) for $a_{\star}(t, s)$ and $a_{\star}(t, s)$ (see (2.11)) as functions of $t$ :

$$
\begin{aligned}
& a_{\star}(t, s)=\widetilde{a}_{\star}(t, s)+\sum_{i=0}^{2 m} z_{i}(t) \widetilde{a}_{i}(s), \widetilde{a}_{i}(s)=\left.\left(\frac{\partial}{\partial t}\right)^{i} a_{\star}(t, s)\right|_{t=1}-\left.\left(\frac{\partial}{\partial t}\right)^{i} a_{\star}(t, s)\right|_{t=0}, \\
& b_{\star}(t, s)=\widetilde{b}_{\star}(t, s)+\sum_{i=0}^{2 m} z_{i}(t) \widetilde{b}_{i}(s), \widetilde{b}_{i}(s)=\left.\left(\frac{\partial}{\partial t}\right)^{i} b_{\star}(t, s)\right|_{t=1}-\left.\left(\frac{\partial}{\partial t}\right)^{i} b_{\star}(t, s)\right|_{t=0} .
\end{aligned}
$$

In accordance to $(5.2)$, the functions $\widetilde{a}_{\star}(t, s)$ and $\widetilde{b}_{\star}(t, s)$ are 1-periodic in $t$. Due to $(2.13)$ it holds $\varphi^{(j)}(0)=\varphi^{(j)}(1)=0$ for $j=1, \ldots, 2 m+1$ implying

$$
\widetilde{a}_{i}(s)=\left[\left.a(1, \varphi(s))\left(\frac{\partial}{\partial t}\right)^{i} \Phi(t, s)^{-\nu}\right|_{t=1}-\left.a(0, \varphi(s))\left(\frac{\partial}{\partial t}\right)^{i} \Phi(t, s)^{-\nu}\right|_{t=0}\right] \varphi^{\prime}(s)^{\mu}
$$

$\mu=(1+\nu) / 2$, and $\widetilde{a}_{i}^{(j)}(0)=\widetilde{a}_{i}^{(j)}(1)=0$ for $j=0, \ldots, 2 m$. After 1 -periodic extension, we have $\widetilde{a}_{i} \in \widetilde{C}^{2 m}(\mathbb{R}), i=0, \ldots, 2 m$, and similarly $\widetilde{b}_{i} \in \widetilde{C}^{2 m}(\mathbb{R})$, $i=0, \ldots, 2 m$. Hence $\widetilde{a}_{\star}, \widetilde{b}_{\star} \in \widetilde{C}^{2 m}(\mathbb{R} \times \mathbb{R})$ (remember that $a_{\star}(t, s)$ and $b_{\star}(t, s)$ are 1-periodic in $s$ ). Denoting by $T_{\star}=A_{\star}+B_{\star}$ the integral operator in (2.10), with $A_{\star}$ and $B_{\star}$ determined by

$$
\left(A_{\star} v\right)(t)=\int_{0}^{1} a_{\star}(t, s) \widetilde{g}(t-s) v(s) d s, \quad\left(B_{\star} v\right)(t)=\int_{0}^{1} b_{\star}(t, s) v(s) d s,
$$

we obtain the decomposition

$$
T_{\star}=\widetilde{A}_{\star}+\widetilde{B}_{\star}+\sum_{i=0}^{2 m} M_{z_{i}}\left(\widetilde{A}_{i}+\widetilde{B}_{i}\right)
$$

where

$$
\begin{aligned}
& \left(\widetilde{A}_{\star} v\right)(t)=\int_{0}^{1} \widetilde{a}_{\star}(t, s) \widetilde{g}(t-s) v(s) d s, \quad\left(\widetilde{B}_{\star} v\right)(t)=\int_{0}^{1} \widetilde{b}_{\star}(t, s) v(s) d s, \\
& \left(\widetilde{A}_{i} v\right)(t)=\int_{0}^{1} \widetilde{g}(t-s) \widetilde{a}_{i}(s) v(s) d s, \quad\left(\widetilde{B}_{i} v\right)(t)=\int_{0}^{1} \widetilde{b}_{i}(s) v(s) d s
\end{aligned}
$$

are periodic integral operators with biperiodic kernels, and the only nonperiodic operators in the decomposition (6.1) are $M_{z_{i}}$ :

$$
\left(M_{z_{i}} v\right)(t)=z_{i}(t) v(t), \quad i=0, \ldots, 2 m
$$

realizing the multiplication with the polynomials $z_{i}$. We approximate the periodic integral operator $\widetilde{A}_{\star}+\widetilde{B}_{\star}$ in (6.1) in analogy to (4.3) by the operator $Q_{2 n}\left(\widetilde{A}_{\star, 2 n}+\widetilde{B}_{\star, 2 n}\right) P_{n}+Q_{n^{\prime}} \widetilde{A}_{\star, n}^{(m)}\left(I-P_{n}\right)$. Also $\widetilde{A}_{i}+\widetilde{B}_{i}$ in (6.1) could be approximated similarly as in (4.3) but since the coefficient functions $\widetilde{a}_{i}(s)$ and 
$\widetilde{b}_{i}(s)$ in $(6.2)$ are independent of $t$, more simple approximations are available: we introduce for $\widetilde{A}_{i}+\widetilde{B}_{i}$ the approximation $\left(\widetilde{A}_{i, n^{\prime}}+\widetilde{B}_{i, n^{\prime}}\right) P_{\left[n^{\prime} / 2\right]}$, where

$$
\left(\widetilde{A}_{i, n^{\prime}} v\right)(t)=\int_{0}^{1} \widetilde{g}(t-s) Q_{n^{\prime}}\left(\widetilde{a}_{i}(s) v(s)\right) d s, \quad \widetilde{B}_{i, n^{\prime}} v=\int_{0}^{1} Q_{n^{\prime}}\left(\widetilde{b}_{i}(s) v(s)\right) d s
$$

and $\left[n^{\prime} / 2\right]$ is the integer part of $n^{\prime} / 2$. Thus we approximate $T_{\star}$ (see $(6.1)$ ) by

$$
\begin{aligned}
T_{\star, n, n^{\prime}}=Q_{2 n}\left(\widetilde{A}_{\star, 2 n}+\widetilde{B}_{\star, 2 n}\right) P_{n} & +Q_{n^{\prime}} \widetilde{A}_{\star, n}^{(m)}\left(I-P_{n}\right) \\
& +\sum_{i=0}^{2 m} M_{z_{i}}\left(\widetilde{A}_{i, n^{\prime}}+\widetilde{B}_{i, n^{\prime}}\right) P_{\left[n^{\prime} / 2\right]} .
\end{aligned}
$$

Given the sample values $v\left(i / n^{\prime}\right), i=0, \ldots, n^{\prime}-1$ of a function $v \in \mathcal{T}_{n^{\prime}}$, the computation of $\left(T_{\star, n, n^{\prime}} v\right)\left(i / n^{\prime}\right), i=0, \ldots, n^{\prime}-1$, costs $O\left(n^{2}\right)$ arithmetical operations (including the assembling of the matrix form of the operator), cf. Section 4. For the operators $T_{\star}$ and $T_{\star, n, n^{\prime}}$, similar estimates as in Lemma 2 hold true.

Lemma 3. Assume (A2), and let $n^{\prime} \sim n^{\tau}, \tau>1$. Then for $1 /(1-\nu)<p<\infty$,

$$
\left\|T_{\star}\left(I-P_{n^{\prime}}\right)\right\|_{\widetilde{L}^{p} \rightarrow C} \leq c n^{-\tau(1-\nu-1 / p)} ;
$$

for $1 /(1-\nu)<p \leq \infty, 0<\lambda<1-\nu-p^{-1}$,

$$
\left\|T_{\star}-T_{\star, n . n^{\prime}}\right\|_{\widetilde{L}^{p} \rightarrow C} \leq c_{p, \lambda}\left(n^{-m}+n^{-\tau \lambda}\right) \log n ;
$$

for $1 /(1-\nu)<p<\infty, 0<\lambda<1-\nu-p^{-1}, v \in \widetilde{W}^{m, p}(\mathbb{R})$,

$$
\begin{gathered}
\left\|T_{\star} v-T_{\star, n . n^{\prime}} v\right\|_{p} \leq c_{p, \lambda}\left(n^{-2 m}+n^{-\tau(m+\lambda)}(\log n)\right)\|v\|_{W^{m, p}}, \\
\left\|T_{\star} v-T_{\star, n . n^{\prime}} v\right\|_{\infty} \leq c_{p, \lambda}\left(n^{-2 m}+n^{-\tau(m+\lambda)}\right)(\log n)\|v\|_{W^{m, p}}
\end{gathered}
$$

and, strengthening (A2) to the assumption (A2'),

$$
\begin{aligned}
\left\|T_{\star} v-T_{\star, n . n^{\prime}} v\right\|_{\infty} & \leq c_{p, \lambda}\left(n^{-2 m-\min \{\mu, 1-\nu-1 / p\}}\right. \\
& \left.+n^{-\tau(m+\lambda)}\right)(\log n)\|v\|_{W^{m, p}} .
\end{aligned}
$$

Proof. By Lemma 2 the counterparts of the estimates (6.4)-(6.7) hold for $A_{\star}+B_{\star}$ and its approximation $Q_{2 n}\left(\widetilde{A}_{\star, 2 n}+\widetilde{B}_{\star, 2 n}\right) P_{n}+Q_{n^{\prime}} \widetilde{A}_{\star, n}^{(m)}\left(I-P_{n}\right)$, and the counterpart of (6.4) holds also for $\widetilde{A}_{i}+\widetilde{B}_{i}$. So we only have to check that the counterparts of estimates $(6.5)-(6.7)$ hold for $\widetilde{A}_{i}+\widetilde{B}_{i}$ and their approximation $\left(\widetilde{A}_{i, n^{\prime}}+\widetilde{B}_{i, n^{\prime}}\right) P_{\left[n^{\prime} / 2\right]}, i=0, \ldots, 2 m$. Split

$$
\widetilde{A}_{i}-\widetilde{A}_{i, n^{\prime}} P_{\left[n^{\prime} / 2\right]}=\widetilde{A}_{i}\left(I-P_{\left[n^{\prime} / 2\right]}\right)+\left(\widetilde{A}_{i}-\widetilde{A}_{i, n^{\prime}}\right) P_{\left[n^{\prime} / 2\right]} .
$$

Consider the first addend in the r.h.s. of (6.8). Let $p$ and $\lambda$ satisfy the conditions $1 /(1-\nu)<p<\infty, 0<\lambda<1-\nu-p^{-1}$. By (4.4), we obtain in support of (6.5)

$$
\left\|\widetilde{A}_{i}\left(I-P_{\left[n^{\prime} / 2\right]}\right)\right\|_{\widetilde{L}^{p} \rightarrow C} \leq c n^{-\tau(1-\nu-1 / p)}=o\left(n^{-\tau \lambda}\right) ;
$$


for $p=\infty$, according to [14] we again obtain

$$
\left\|\widetilde{A}_{i}\left(I-P_{\left[n^{\prime} / 2\right]}\right)\right\|_{\widetilde{L}^{\infty} \rightarrow C} \leq c n^{-\tau(1-\nu)} \log n=o\left(n^{-\tau \lambda}\right) .
$$

For $v \in \widetilde{W}^{m, p}(\mathbb{R})$, due to (4.14) we obtain in support of (6.6)-(6.7)

$$
\begin{gathered}
\left\|\widetilde{A}_{i}\left(I-P_{\left[n^{\prime} / 2\right]}\right) v\right\|_{\infty} \leq c\left\|\widetilde{A}_{i}\left(I-P_{\left[n^{\prime} / 2\right]}\right)\right\|_{\widetilde{L}^{p} \rightarrow C}\left\|\left(I-P_{\left[n^{\prime} / 2\right]}\right) v\right\|_{p} \\
\leq c^{\prime} n^{-\tau(1-\nu-1 / p)} n^{-\tau m}\|v\|_{W^{m, p}}=o\left(n^{-\tau(m+\lambda)}\|v\|_{W^{m, p}} .\right.
\end{gathered}
$$

Consider the second addend in the r.h.s of (6.8). We have with any $w \in \mathcal{T}_{\left[n^{\prime} / 2\right]}$, $p^{-1}+q^{-1}=1$ that

$$
\begin{aligned}
& \left(\left(\widetilde{A}_{i}-\widetilde{A}_{i, n^{\prime}}\right) P_{\left[n^{\prime} / 2\right]} v\right)(t)=\int_{0}^{1} \widetilde{g}(t-s)\left(I-Q_{n^{\prime}}\right)\left(\left(\widetilde{a}_{i}(s)-w(s)\right)\left(P_{\left[n^{\prime} / 2\right]} v\right)(s)\right) d s, \\
& \left\|\left(\widetilde{A}_{i}-\widetilde{A}_{i, n^{\prime}}\right) P_{\left[n^{\prime} / 2\right]} v\right\|_{\infty} \leq\|\widetilde{g}\|_{q}\left\|\left(I-Q_{n^{\prime}}\right)\left(\widetilde{a}_{i}-w\right) P_{\left[n^{\prime} / 2\right]} v\right\|_{p} \\
& \quad \leq c\left\|\widetilde{a}_{i}-w\right\|_{\infty}\left\|P_{\left[n^{\prime} / 2\right]} v\right\|_{\infty} .
\end{aligned}
$$

This supports estimates (6.4)-(6.7) since

$$
\left\|P_{\left[n^{\prime} / 2\right]} v\right\|_{\infty} \leq n\|v\|_{1},\left\|P_{\left[n^{\prime} / 2\right]} v\right\|_{\infty} \leq c_{p}\|v\|_{W^{m, p}}
$$

and for $\widetilde{a}_{i} \in \widetilde{C}^{2 m}(\mathbb{R})$, there exists a $w \in \mathcal{T}_{\left[n^{\prime} / 2\right]}$ such that $\left\|\widetilde{a}_{i}-w\right\|_{\infty} \leq c n^{-2 \tau m}$.

In the equality $\bar{u}=T_{\star} \widetilde{u}+\bar{f}$ which is a short writing of the integral relation (2.10), we approximate the solution $\widetilde{u}$ of (2.6) by the solution $\widetilde{u}_{n, n^{\prime}}$ of (4.2), and we approximate the integral operator $(6.1)$ by $(6.3)$, obtaining

$$
\bar{u}_{n, n^{\prime}}:=T_{\star, n . n^{\prime}} \widetilde{u}_{n, n^{\prime}}+\bar{f},
$$

which we treat as an approximate solution of equation (2.5). The approximation $\bar{u}_{n, n^{\prime}}$ is not discrete. Its discrete counterpart will be introduced and discussed in Section 7.

Theorem 2. Assume the conditions of Theorem 1. Then

$$
\begin{aligned}
& \left\|\bar{u}-\bar{u}_{n, n^{\prime}}\right\|_{\infty} \leq c n^{-2 m}(\log n)\|\widetilde{f}\|_{C^{m}}, \\
& \left\|\bar{u}-\bar{u}_{n, n^{\prime}}\right\|_{p} \leq c_{p} n^{-2 m}\|\widetilde{f}\|_{C^{m}}, \quad 1 \leq p<\infty
\end{aligned}
$$

where $\bar{u}(t)=u(\varphi(t))$ is the solution of equation (2.5), $u$ is the solution of equation (1.1), and $\bar{u}_{n, n^{\prime}}$ is defined by (6.9) in which $\widetilde{u}_{n, n^{\prime}}$ is the solution of equation (4.2). If $a$ and $b$ satisfy $\left(\mathrm{A} 2^{\prime}\right)$ then

$$
\left\|\bar{u}-\bar{u}_{n, n^{\prime}}\right\|_{\infty}=o\left(n^{-2 m}\right)\|\widetilde{f}\|_{C^{m}}
$$

i.e. $\left\|\bar{u}-\bar{u}_{n, n^{\prime}}\right\|_{\infty} n^{2 m} \rightarrow 0 \quad$ as $n \rightarrow \infty$. 
Proof. Since

$$
\begin{aligned}
& \bar{u}-\bar{u}_{n, n^{\prime}}=T_{\star} \widetilde{u}-T_{\star, n . n^{\prime}} \widetilde{u}_{n, n^{\prime}} \\
& \quad=T_{\star}\left(\widetilde{u}-\widetilde{u}_{n, n^{\prime}}\right)+\left(T_{\star, n \cdot n^{\prime}}-T_{\star}\right)\left(\widetilde{u}-\widetilde{u}_{n, n^{\prime}}\right)+\left(T_{\star} \widetilde{u}-T_{\star, n . n^{\prime}} \widetilde{u}\right),
\end{aligned}
$$

we have

$$
\begin{aligned}
& \left\|\bar{u}-\bar{u}_{n, n^{\prime}}\right\|_{\infty} \leq\left\|T_{\star}\left(\widetilde{u}-\widetilde{u}_{n, n^{\prime}}\right)\right\|_{\infty}+\left\|T_{\star}-T_{\star, n \cdot n^{\prime}}\right\|_{\widetilde{L}^{p} \rightarrow C}\left\|\widetilde{u}-\widetilde{u}_{n, n^{\prime}}\right\|_{p} \\
& \quad+\left\|T_{\star} \widetilde{u}-T_{\star, n \cdot n^{\prime}} \widetilde{u}\right\|_{\infty} .
\end{aligned}
$$

Due to (4.1), $(1+\tau) m>2 m$; taking $p<\infty$ sufficiently large and $\lambda>0$ sufficiently close to $1-\nu-p^{-1}$, we have $\tau(m+\lambda)>2 m$, and the estimates (6.5), (4.11) imply for $1 /(1-\nu)<p<\infty$ that

$$
\begin{aligned}
\| T_{\star} & -T_{\star, n . n^{\prime}}\left\|_{\widetilde{L}^{p} \rightarrow C}\right\| \widetilde{u}-\widetilde{u}_{n, n^{\prime}} \|_{p} \leq c\left(n^{-m}+n^{-\tau \lambda}\right) \\
& \times n^{-\tau m}(\log n)^{2}\|\widetilde{f}\|_{W^{m, p}}=o\left(n^{-2 m}\right)\|\widetilde{f}\|_{W^{m, p}},
\end{aligned}
$$

whereas (6.6)-(6.7) yield

$$
\begin{aligned}
& \left\|T_{\star} \widetilde{u}-T_{\star, n . n^{\prime}} \widetilde{u}\right\|_{p} \leq c_{p} n^{-2 m}\|\widetilde{f}\|_{W^{m, p}}, \quad 1 /(1-\nu)<p<\infty, \\
& \left\|T_{\star} \widetilde{u}-T_{\star, n . n^{\prime}} \widetilde{u}\right\|_{\infty} \leq c n^{-2 m}(\log n)\|\widetilde{f}\|_{W^{m, p}}
\end{aligned}
$$

and in the case of $\left(\mathrm{A} 2^{\prime}\right)$,

$$
\left\|T_{\star} \widetilde{u}-T_{\star, n . n^{\prime}} \widetilde{u}\right\|_{\infty}=o\left(n^{-2 m}\right)\|\widetilde{f}\|_{W^{m, p}} .
$$

Below we show that

$$
\begin{aligned}
& \left\|T_{\star}\left(\widetilde{u}-\widetilde{u}_{n, n^{\prime}}\right)\right\|_{\infty} \leq c n^{-2 m}\|\widetilde{f}\|_{C^{m}} \text { if } a, b \in C^{2 m}([0,1] \times[0,1]), \\
& \left\|T_{\star}\left(\widetilde{u}-\widetilde{u}_{n, n^{\prime}}\right)\right\|_{\infty}=o\left(n^{-2 m}\right)\|\widetilde{f}\|_{C^{m}} \text { if } a, b \in \mathcal{H}^{2 m, \mu}([0,1] \times[0,1]) .
\end{aligned}
$$

Now the estimates (6.10)-(6.12) immediately follow from (6.13)-(6.17); actually, we obtain $(6.11)$ only for $1 /(1-\nu)<p<\infty$; to obtain (6.11) for smaller $p \in[1,1 /(1-\nu)]$ we exploit the monotonicity in $p$ of the norm $\|\cdot\|_{p}$.

It remains to prove (6.17). Applying to both sides of equality (4.13) the operator $T_{\star}$ and using the equality $\left(I-\widetilde{T}_{n, n^{\prime}}\right)^{-1}=I+\left(I-\widetilde{T}_{n, n^{\prime}}\right)^{-1} \widetilde{T}_{n, n^{\prime}}$, we obtain

$$
\begin{aligned}
& T_{\star}\left(\widetilde{u}-\widetilde{u}_{n, n^{\prime}}\right)=T_{\star}\left(\widetilde{f}-P_{n^{\prime}} \tilde{f}\right)+T_{\star}\left(\widetilde{T}-\widetilde{T}_{n, n^{\prime}}\right) \widetilde{u} \\
& \quad+T_{\star}\left(I-\widetilde{T}_{n, n^{\prime}}\right)^{-1}\left(\widetilde{T}_{n, n^{\prime}}\left(\widetilde{f}-P_{n^{\prime}} \widetilde{f}\right)+\widetilde{T}_{n, n^{\prime}}\left(\widetilde{T}-\widetilde{T}_{n, n^{\prime}}\right) \widetilde{u}\right) \\
& \quad\left\|T_{\star}\left(\widetilde{u}-\widetilde{u}_{n, n^{\prime}}\right)\right\|_{\infty} \leq\left\|T_{\star}\left(\widetilde{f}-P_{n^{\prime}} \widetilde{f}\right)\right\|_{\infty}+c_{1}\left\|\widetilde{T}_{n, n^{\prime}}\left(\widetilde{f}-P_{n^{\prime}} \widetilde{f}\right)\right\|_{\infty} \\
& \quad+c_{2}\left\|\left(\widetilde{T}-\widetilde{T}_{n, n^{\prime}}\right) \widetilde{u}\right\|_{p},
\end{aligned}
$$

where we took into account (4.12), the boundedness of $T_{\star}: \widetilde{L}^{p} \rightarrow C$, and the uniform boundedness of operators $\widetilde{T}_{n, n^{\prime}}: \widetilde{L}^{p} \rightarrow \widetilde{C}, n \in \mathbb{N}$ (see (4.5)). 
Using the equality $I-P_{n^{\prime}}=\left(I-P_{n^{\prime}}\right)^{2}$, (6.4) and (4.14), we estimate

$$
\begin{aligned}
& \left.\left\|T_{\star}\left(\tilde{f}-P_{n^{\prime}} \tilde{f}\right)\right\|_{\infty} \leq\left\|T_{\star}\left(I-P_{n^{\prime}}\right)\right\|_{\widetilde{L}^{p} \rightarrow C} \| \widetilde{f}-P_{n^{\prime}} \tilde{f}\right) \|_{p} \\
& \quad \leq c n^{-\tau(1-\nu-1 / p)} n^{-\tau m}\|\widetilde{f}\|_{W^{m, p}}=c n^{-\tau(m+1-\nu-1 / p)}\|\widetilde{f}\|_{W^{m, p}} .
\end{aligned}
$$

According to (4.1), $\tau(m+1-\nu)>2 m$, for sufficiently large $p$ also $\tau(m+1-$ $\nu-1 / p)>2 m$, hence

$$
\left\|T_{\star}\left(\widetilde{f}-P_{n^{\prime}} \widetilde{f}\right)\right\|_{\infty}=o\left(n^{-2 m}\right)\|\widetilde{f}\|_{C^{m}} .
$$

With the help of (6.5) and (4.14) we get similarly as in (6.14), (6.19)

$$
\begin{gathered}
\left\|\widetilde{T}_{n, n^{\prime}}\left(\widetilde{f}-P_{n^{\prime}} \widetilde{f}\right)\right\|_{\infty} \leq\left\|\widetilde{T}_{n, n^{\prime}}-\widetilde{T}\right\|_{\widetilde{L}^{p} \rightarrow C}\left\|\widetilde{f}-P_{n^{\prime}} \widetilde{f}\right\|_{p} \\
+\left\|\widetilde{T}\left(\tilde{f}-P_{n^{\prime}} \widetilde{f}\right)\right\|_{\infty}=o\left(n^{-2 m}\right)\|\widetilde{f}\|_{C^{m}} .
\end{gathered}
$$

Similarly to $(6.15),(6.16)$ we have

$$
\begin{aligned}
& \left\|\widetilde{T} \widetilde{u}-\widetilde{T}_{n . n^{\prime}} \widetilde{u}\right\|_{p} \leq c_{p} n^{-2 m}\|\widetilde{f}\|_{W^{m, p}}, \quad 1 /(1-\nu)<p<\infty, \\
& \left\|\widetilde{T} \widetilde{u}-\widetilde{T}_{n \cdot n^{\prime}} \widetilde{u}\right\|_{\infty}=o\left(n^{-2 m}\right)\|\widetilde{f}\|_{C^{m}} \text { if } a, b \in \mathcal{H}^{2 m, \mu}([0,1] \times[0,1]) .
\end{aligned}
$$

Plugging (6.19)-(6.20) into (6.18) we obtain (6.17).

Remark 4. Consider the case where we use some approximation $\tilde{f}_{n, n^{\prime \prime}}$ of $\tilde{f}$ to compute the Fourier coefficients of $\tilde{f}$ as in Remark 3 so that (4.17) and hence also (4.18) hold true. Then instead of (6.9) we obtain the approximation $\bar{u}_{n, n^{\prime}, n^{\prime \prime}}=T_{\star, n . n^{\prime}} \widetilde{u}_{n, n^{\prime}, n^{\prime \prime}}+\bar{f}$. Following the proof scheme of Theorem 2 it is easily seen that, under conditions of Theorem 1 ,

$$
\begin{aligned}
& \left\|\bar{u}_{n, n^{\prime}}-\bar{u}_{n, n^{\prime}, n^{\prime \prime}}\right\|_{\infty} \leq c n^{-2 m}\|\widetilde{f}\|_{C^{m}}, \\
& \left\|\bar{u}_{n, n^{\prime}}-\bar{u}_{n, n^{\prime}, n^{\prime \prime}}\right\|_{\infty} \leq c n^{-2 m}\|\tilde{f}\|_{C^{m}} \text { if } a, b \text { satisty }\left(A 2^{\prime}\right) .
\end{aligned}
$$

Hence estimates $(6.10)-(6.12)$ remain to be valid also for $\bar{u}_{n, n^{\prime}, n^{\prime \prime}}$.

Remark 5. The operator $\widetilde{T}$ has the property (compare (3.2) in [14]) that for $\frac{1}{1-\nu}<p \leq \infty, 0<\lambda<1-\nu-\frac{1}{p}, \widetilde{T}: \widetilde{W}^{m, p}(\mathbb{R}) \rightarrow \widetilde{\mathcal{H}}^{m, \lambda}(\mathbb{R})$ is bounded; moreover, for those $p$ and $\lambda$, it can be proved by the techniques of [14] that $\left\|\widetilde{T}-\widetilde{T}_{n, n^{\prime}}\right\|_{\widetilde{W}^{m, p}(\mathbb{R}) \rightarrow \widetilde{\mathcal{H}}^{m, \lambda}(\mathbb{R})} \rightarrow 0$ as $n \rightarrow \infty$. The definitions (6.1) and (6.3) of $T_{\star}$ and $T_{\star, n, n^{\prime}}$ enable to extend these relations: for $\frac{1}{1-\nu}<p \leq \infty, 0<\lambda<$ $1-\nu-1 / p, T_{\star}: \widetilde{W}^{m, p}(\mathbb{R}) \rightarrow \mathcal{H}^{m, \lambda}[0,1]$ is bounded,

$$
\left\|T_{\star}-T_{\star, n, n^{\prime}}\right\|_{\widetilde{W}^{m, p}(\mathbb{R}) \rightarrow \mathcal{H}^{m, \lambda}} \rightarrow 0 \text { as } n \rightarrow \infty .
$$

From here we conclude for the solution $\widetilde{u}_{n, n^{\prime}}$ of equation (4.2) that

$$
\left\|\widetilde{u}_{n, n^{\prime}}\right\|_{W^{m, p}} \leq c_{p}\left\|P_{n} \widetilde{f}\right\|_{W^{m, p}} \leq c_{p}^{\prime}\|\widetilde{f}\|_{W^{m, p}}, \frac{1}{1-\nu}<p<\infty
$$




$$
\left\|T_{\star, n, n^{\prime}} \widetilde{u}_{n, n^{\prime}}\right\|_{\mathcal{H}^{m, \lambda}} \leq c_{p, \lambda}\|\widetilde{f}\|_{W^{m, p}}, \frac{1}{1-\nu}<p<\infty, \quad 0<\lambda<1-\nu-\frac{1}{p} .
$$

Deriving (6.21) we exploited the fact that $\left\|P_{n}\right\|_{\widetilde{W}^{m, p}(\mathbb{R}) \rightarrow \widetilde{W}^{m, p}(\mathbb{R})} \leq c_{p}$ for $n \in \mathbb{N}$, $1<p<\infty$. Inequality (6.22) will be helpful in Section 7 . It is valid also if we replace $\widetilde{u}_{n, n^{\prime}}$ by $\widetilde{u}_{n, n^{\prime}, n^{\prime \prime}}$ assuming the conditions of Remark 3 .

\section{$7 \quad\left(C, C^{m}\right)$ fast/quasifast solver of equation (1.1)}

According to Section 4, the sample values $\widetilde{u}_{n, n^{\prime}}\left(i / n^{\prime}\right), i=0, \ldots, n^{\prime}-1$ of the solution $\widetilde{u}_{n, n^{\prime}} \in \mathcal{T}_{n^{\prime}}$ to $(4.2)$ are available at the cost of $O\left(n^{2}\right)$ arithmetical operations. After that also the values $\left(T_{\star, n, n^{\prime}} \widetilde{u}_{n, n^{\prime}}\right)\left(i / n^{\prime}\right), i=0, \ldots, n^{\prime}$ of $T_{\star, n, n^{\prime}} \widetilde{u}_{n, n^{\prime}}$ can be computed in $O\left(n^{2}\right)$ arithmetical operations, and we can use the local (algebraic) polynomial interpolation to approximate $T_{\star, n, n^{\prime}} \widetilde{u}_{n, n^{\prime}}$ between the grid points $i / n^{\prime}, i=0, \ldots, n^{\prime}$. Define the polynomially interpolated version $\bar{v}_{n, n^{\prime}}(t)$ of $\bar{u}_{n, n^{\prime}}=T_{\star, n, n^{\prime}} \widetilde{u}_{n, n^{\prime}}+\bar{f}$ (see (6.9) and Section 3.4) as

$$
\bar{v}_{n, n^{\prime}}=\Pi_{n^{\prime}, m+1} T_{\star, n, n^{\prime}} \widetilde{u}_{n, n^{\prime}}+\Pi_{n^{2}, m} \bar{f},
$$

where $\Pi_{n^{\prime}, m+1}$ is an operator of local interpolation of functions given on the grid $\left\{i / n^{\prime}: i=0, \ldots, n^{\prime}\right\}$ by polynomials of degree $m$ (or of order $m+1$ ) and $\Pi_{n^{2}, m}$ is an operator of local interpolation by polynomials of degree $m-1$ from the grid values on the net $\left.\left\{i / n^{2}\right): i=0, \ldots, n^{2}\right\}$. Thus, the unknown parameters $\left(T_{\star, n, n^{\prime}} \widetilde{u}_{n, n^{\prime}}\right)\left(i / n^{\prime}\right), i=0, \ldots, n^{\prime}$ of $\bar{v}_{n, n^{\prime}}$ are available at the cost of $O\left(n^{2}\right)$ arithmetical operations, and having them in hand, the value of $\bar{v}_{n, n^{\prime}}$ at any point $t \in[0,1]$ is available at the cost of $O(1)$ arithmetical operations.

We could define the approximate solution of equation (1.1) by the formula

$$
v_{n, n^{\prime}}(x)=\bar{v}_{n, n^{\prime}}\left(\varphi^{-1}(x)\right), \quad 0 \leq x \leq 1,
$$

with $\varphi^{-1}$, the inverse of $\varphi$ (see $(2.4)$ ), but since we cannot present a closed formula for $\varphi^{-1}(x)$ we first approximate it by a suitable local interpolation. Let us precompute $x_{i, n}=\varphi(i / n)$ for $i=0, \ldots, n$ (this costs altogether only $O(n)$ arithmetical operations) and use them to approximate $t=\varphi^{-1}(x)$ for any given $x \in[0,1]$ via a local interpolation by polynomials of degree $2 m-1$. Note that $\varphi^{-1} \in C^{2 m, 1 / r}(0,1)$ where $r$ is the (smoothing) parameter in the definition of $\varphi$ (actually $\varphi^{-1} \in C^{l, 1 / r}(0,1)$ for any $l \in \mathbb{N}$ ), and $\left\{x_{i, n}: i=0, \ldots, n\right\}$ is a suitable graded grid for the interpolation of such functions - denoting by $\psi_{n}$ the interpolation approximation of $\psi=\varphi^{-1}$, we have

$$
\left|\varphi^{-1}(x)-\psi_{n}(x)\right| \leq c n^{-2 m}\left\|\varphi^{-1}\right\|_{C^{2 m, 1 / r}}, \quad 0 \leq x \leq 1,
$$

see, e.g., [26]. Instead of (7.2), we define the final approximation to the solution of equation (1.1) via the formula

$$
u_{n, n^{\prime}}(x)=\bar{v}_{n, n^{\prime}}\left(\psi_{n}(x)\right), \quad 0 \leq x \leq 1 .
$$

The grid values $\left(T_{\star, n, n^{\prime}} \widetilde{u}_{n, n^{\prime}}\right)\left(i / n^{\prime}\right)\left(i=0, \ldots, n^{\prime}\right)$ and $\bar{f}\left(i / n^{2}\right)=f\left(\varphi\left(i / n^{2}\right)\right)(i=$ $\left.0, \ldots, n^{2}\right)$ can be considered as the parameters of the approximate solution 
(7.4). The grid values of $f$ belong to a given information whereas the grid values of $T_{\star, n, n^{\prime}} \widetilde{u}_{n, n^{\prime}}$ are available at the cost of $O\left(n^{2}\right)$ arithmetical operations using $n_{\star}=O\left(n^{2}\right)$ sample values of $a$ and $b$ and the $n^{2}+1$ sample values of $f$ just listed. To see that with (7.4) we have designed a fast/quasifast solver of equation (1.1), it remains to establish for $u_{n, n^{\prime}}$ the estimates of type $(1.2) /(1.3)$. Estimates (7.5)-(7.6) (see Theorem 3 below) mean that under condition $a, b \in$ $C^{2 m}([0,1] \times[0,1])$ approximation $(7.4)$ defines a $\left(C, C^{m, \nu}\right)$-quasifast solver of equation (1.1) which is $\left(L^{p}, C^{m, \nu}\right)$-fast for $1 \leq p<\infty$ and, moreover, this solver is even $\left(C, C^{m, \nu}\right)$-fast if the $m$ th derivatives of $a, b$ are Hoelder continuous.

Theorem 3. Assume the conditions of Theorem 1. Then we have for all sufficiently large $n \in \mathbb{N}$ that

$$
\begin{aligned}
& \left\|u-u_{n, n^{\prime}}\right\|_{\infty} \leq c n^{-2 m}(\log n)\|f\|_{C^{m, \nu}}, \\
& \left\|u-u_{n, n^{\prime}}\right\|_{p} \leq c_{p} n^{-2 m}\|f\|_{C^{m, \nu}}, \quad 1 \leq p<\infty,
\end{aligned}
$$

where $u$ is the solution of (1.1) and $u_{n, n^{\prime}}$ is defined by (7.1), (7.4) with the solution $\widetilde{u}_{n, n^{\prime}}$ of equation (4.2). The full computation cost of $u_{n, n^{\prime}}$ is $O\left(n^{2}\right)$ flops. So we have constructed a $\left(C, C^{m, \nu}\right)$-quasifast solver of equation (1.1); this solver is $\left(L^{p}, C^{m, \nu}\right)$-fast. Moreover,. under condition $\left(\mathrm{A} 2^{\prime}\right)$ this solver is $\left(C, C^{m, \nu}\right)-$ fast:

$$
\left\|u-u_{n, n^{\prime}}\right\|_{\infty} \leq c n^{-2 m}\|f\|_{C^{m, \nu}} .
$$

Proof. Let the conditions of Theorem 1 be fulfilled. With $x=\varphi(t), 0 \leq t \leq 1$, we have for $u$, the solution of equation (1.1), and for its approximation $u_{n, n^{\prime}}$, defined by (7.1) and (7.4), that

$$
\begin{aligned}
& u(x)-u_{n, n^{\prime}}(x)=u(\varphi(t))-\bar{v}_{n, n^{\prime}}\left(\psi_{n}(x)\right) \\
& \quad=\left[\bar{u}(t)-\bar{u}_{n, n^{\prime}}(t)\right]+\left[\bar{u}_{n, n^{\prime}}(t)-\bar{v}_{n, n^{\prime}}(t)\right]+\left[\bar{v}_{n, n^{\prime}}\left(\varphi^{-1}(x)\right)-\bar{v}_{n, n^{\prime}}\left(\psi_{n}(x)\right)\right] .
\end{aligned}
$$

We obtain the claims $(7.5)-(7.6)$ estimating $\bar{u}-\bar{u}_{n, n^{\prime}}$ by $(6.10)-(6.12)$, respectively, and noticing that in accordance to (7.1), (7.3) and (6.22)

$$
\begin{gathered}
\max _{0 \leq x \leq 1}\left|\bar{v}_{n, n^{\prime}}\left(\varphi^{-1}(x)\right)-\bar{v}_{n, n^{\prime}}\left(\psi_{n}(x)\right)\right| \leq \sup _{0 \leq t \leq 1}\left|\bar{v}_{n, n^{\prime}}^{\prime}(t)\right|\left\|\varphi^{-1}-\psi_{n}\right\|_{\infty} \\
\quad \leq c\left(\left\|T_{\star, n, n^{\prime}} \widetilde{u}_{n, n^{\prime}}\right\|_{C^{1}}+\|\bar{f}\|_{C^{1}}\right) n^{-2 m} \leq c^{\prime} n^{-2 m}\|f\|_{C^{m, \nu}}
\end{gathered}
$$

and that in accordance to (6.9), (7.1) and (6.22) with sufficiently large $p<\infty$ and $\lambda$ close to $1-\nu$,

$$
\begin{aligned}
& \left\|u_{n, n^{\prime}}-v_{n, n^{\prime}}\right\|_{\infty} \leq\left\|\left(I-\Pi_{n^{2}, m}\right) \bar{f}\right\|_{\infty}+\left\|\left(I-\Pi_{n^{\prime}, m+1}\right) T_{\star, n, n^{\prime}} \widetilde{u}_{n, n^{\prime}}\right\|_{\infty} \\
& \leq c\left(n^{2}\right)^{-m}\left\|\bar{f}^{(m)}\right\|_{\infty}+c\left(n^{\prime}\right)^{-(m+\lambda)}\left\|T_{\star, n, n^{\prime}} \widetilde{u}_{n, n^{\prime}}\right\|_{\mathcal{H}^{m, \lambda}} \leq c^{\prime} n^{-2 m}\|f\|_{C^{m, \nu}} .
\end{aligned}
$$

Introducing a more dense basic interpolation set than $\left\{x_{i, n}: i=0, \ldots, n\right\}$ exploited above we obtain more accurate approximation of $\varphi^{-1}$ rather than (7.3). 
For instance, the node set $\left\{x_{i, n}=\varphi\left(i / n^{2}\right): i=0, \ldots, n^{2}\right\}$ is still computable in $O\left(n^{2}\right)$ arithmetical operations and leads to the estimate (cf. (7.3))

$$
\left\|\varphi^{-1}-\psi_{n}\right\|_{\infty} \leq c n^{-4 m}\left\|\varphi^{-1}\right\|_{C^{2 m, 1 / r}}
$$

In the following remark we assume quite moderate strengthening of (7.3). Remark 6. Assume $\left(A 2^{\prime}\right)$ and let $\left\|\varphi^{-1}-\psi_{n}\right\|_{\infty}=o\left(n^{-2 m}\right)$. Then (cf. (7.6))

$$
\left\|u-u_{n, n^{\prime}}\right\|_{\infty} \leq c\left\|\left(I-\Pi_{n^{2}, m}\right) \bar{f}\right\|_{\infty}+o\left(n^{-2 m}\right)\|f\|_{C^{m, \nu}} .
$$

Thus, the main part of the error $u-u_{n, n^{\prime}}$ is caused by the interpolation of $\bar{f}$.

\section{Some open problems for future study}

The main purpose of the present paper has been to construct a fast/quasifast solver for a nonperiodical weakly singular integral equation of the form (1.1). To this end we have reduced the original problem to a periodic problem.

It seems that Theorem 3 remains partly valid when $P_{n^{\prime}} \widetilde{f}$ in $(4.2)$ is replaced by $Q_{n^{\prime}} \tilde{f}$. Note that this modified solver has computational advantages compared with (4.2) since the grid values of $\widetilde{f}$ and $Q_{n^{\prime}} \widetilde{f}$ are known.

There also arises a question about extension of the obtained results to more general equations rather than (1.1). In particular, is it possible to modify the proposed construction of $\left(C, C^{m, \nu}\right)$-fast/quasifast solvers for equations (1.1) so that also some boundary singularities of $a(x, y)$ and $b(x, y)$ are allowed? Moreover, it is useful to extend the results of the paper to the case where $a(x, y)$ and $b(x, y)$ may have jumps on the diagonal $x=y$ of the square $0 \leq x, y \leq 1$ but are smooth in upper and lower triangles. In particular, can the results be extended to the case of weakly singular Volterra integral equations? We hope to answer these questions elsewhere.

\section{Acknowledgements}

This work was supported by institutional research funding IUT20-57 of the Estonian Ministry of Education and Research.

\section{References}

[1] K. Atkinson and W. Han. Theoretical Numerical Analysis. Springer, New York, 2009 .

[2] W. Dahmen, H. Harbrecht and R. Schneider. Compression techniques for boundary integral equations-asymptotically optimal complexity estimates. SIAM J. Numer. Anal, 43:2251-2271, 2006. https://doi.org/10.1137/S0036142903428852.

[3] T. Diogo, P. M. Lima, A. Pedas and G. Vainikko. Smoothing transformation and spline collocation for weakly singular Volterra integro-differential equations. Appl. Numer. Math, 114:63-76, 2017. 
[4] K. V. Emel'yanov and A. M. Il'in. The number of arithmetical operations necessary for the approximate solution of Fredholm integral equations. USSR Comput. Math. Phys, 7:259-267, 1967. https://doi.org/10.1016/0041-5553(67)90160-7. (In Russian)

[5] K. Frank, S. Heinrich and S. Pereverzev. Information complexity of multivariate Fredholm integral equations in Sobolev classes. J. Complexity, 12:17-34, 1996. https://doi.org/10.1006/jcom.1996.0004.

[6] I. G. Graham. Singularity expansions for the solutions of second kind Fredholm integral equations with weakly singular convolution kernels. J. Integral Equations, 4:1-30, 1982.

[7] M. Kolk, A. Pedas and G. Vainikko. High-order methods for Volterra integral equations with general weak singularities. Numer. Funct. Anal. Optim, 30(10):1002-1024, 2009. https://doi.org/10.1080/01630560903393154.

[8] G. Monegato and L. Scuderi. High order methods for weakly singular integral equations with nonsmooth input functions. Math. Comput, 67:1493-1515, 1998. https://doi.org/10.1090/S0025-5718-98-01005-9.

[9] O. Nevanlinna. Convergence of iterations for linear equations. Birkhäuser, Basel, 1993. https://doi.org/10.1007/978-3-0348-8547-8.

[10] K. Orav-Puurand, A. Pedas and G. Vainikko. Central part interpolation schemes for integral equations with singularities. J. Int. Eq. Appl, 29(3):401-440, 2017. https://doi.org/10.1216/JIE-2017-29-3-401.

[11] I. Parts, A. Pedas and E. Tamme. Piecewise polynomial collocation for Fredholm integro-differential equations with weakly singular kernels. SIAM J. Numer. Anal, 43:1897-1911, 2005. https://doi.org/10.1137/040612452.

[12] A. Pedas and G. Vainikko. Integral equations with diagonal and boundary singularities of the kernel. ZAA, 25:487-516, 2006. https://doi.org/10.4171/ZAA/1304.

[13] A. Pedas and G. Vainikko. Smoothing transformation and piecewise polynomial projection methods for weakly singular Fredholm integral equations. Comm. Pure and Appl. Anal, 5:395-413, 2006. https://doi.org/10.3934/cpaa.2006.5.395.

[14] A. Pedas and G. Vainikko. What is the complexity of periodic weakly singular integral equations? BIT Num. Math, 48:315-335, 2008. https://doi.org/10.1007/s10543-008-0181-0.

[15] A. Pedas and G. Vainikko. On the regularity of solutions to integral equations with nonsmooth kernels on a union of open intervals. J. Comp. Appl. Math, 229:440-451, 2009. https://doi.org/10.1016/j.cam.2008.04.009.

[16] S. V. Pereverzev. On the complexity of the problem of finding the solutions of Fredholm equations of the second kind with smooth kernels. I. Ukrain. Math. Zh, 40(1):71-76, 1988. https://doi.org/10.1007/bf01056451.

[17] S. V. Pereverzev. On the complexity of the problem of finding the solutions of Fredholm equations of the second kind with smooth kernels. II. Ukrain. Math. Zh, 41(2):169-173, 1989. https://doi.org/10.1007/BF01060382.

[18] S. V. Pereverzev. Hyperbolic cross and the complexity of the approximate solution of Fredholm integral equations of the second kind with differentiable kernels. Sibirsk. Math. Zh, 32(1):85-92, 1991. https://doi.org/10.1007/BF00970164. 
[19] S. V. Pereverzev and K. Sh. Makhkamov. Information complexity of weakly singular integral equations. Ukrainian Math. J, 46:1527-1533, 1994. https://doi.org/10.1007/BF01058886. (In Russian)

[20] J. Pitkäranta. Estimates for the derivatives of solutions to weakly singular Fredholm integral equations. SIAM J. Math. Anal, 11:952-968, 1980. https://doi.org/10.1137/0511085.

[21] J. Saranen and G. Vainikko. Periodic Integral and Pseudodifferential Equations with Numerical Approximation. Springer, Berlin, 2002. https://doi.org/10.1007/978-3-662-04796-5.

[22] J. F. Traub, G. Wozniakowski and H. Wozniakowski. A General Theory of Optimal Algorithms. Academic Press, New York, 1980.

[23] J. F. Traub, G. Wozniakowski and H. Wozniakowski. Information-Based Complexity. American Press, Boston, 1988.

[24] L. N. Trefethen and D. Bau. Numerical Linear Algebra. SIAM, Philadelphia, 1997. https://doi.org/10.1137/1.9780898719574.

[25] E. Vainikko and G. Vainikko. A spline product quasi-interpolation method for weakly singular Fredholm integral equations. SIAM J. Numer. Anal, 46:17991820, 2008. https://doi.org/10.1137/070693308.

[26] G. Vainikko. Multidimensional Weakly Singular Integral Equations. SpringerVerag, Berlin, 1993. https://doi.org/10.1007/BFb0088979.

[27] G. Vainikko. GMRES and discrete approximation of operators. Proc. Estonian Acad Sci. Phys. Math, 53:124-131, 2004.

[28] G. Vainikko. Fast solvers of integral equations of the second kind: quadrature methods. J. Integr. Eq. Appl., 17:91-120, 2005. https://doi.org/10.1216/jiea/1181075312.

[29] G. Vainikko. Fast solvers of integral equations. Lecture notes, HUT, UT, 2006. http://www.ut.ee/ gen/FASTlecturesSIAM.pdf

[30] G. Vainikko. Fast wavelet solvers of periodic integral equations. J. Analysis, 14:243-265, 2006.

[31] G. Vainikko, A. Kivinukk and J. Lippus. Fast solvers of integral equations of the second kind: wavelet methods. J. Complexity, 21:243-273, 2005. https://doi.org/10.1016/j.jco.2004.07.002.

[32] G. Vainikko and A. Pedas. The properties of solutions of weakly singular integral equations. J. Austral. Math. Soc, Ser.B, 22:419-430, 1980.

[33] G. Vainikko and I. Zolk. Fast spline quasicollocation solvers of integral equations. Math. Modelling and Analysis, 12:515-538, 2007. https://doi.org/10.3846/13926292.2007.12.515-538.

[34] A. G. Werschulz. Where does smoothness count the most for Fredholm equations of the second kind with noisy information? J. Complexity, 19:758-798, 2003. https://doi.org/10.1016/S0885-064X(03)00030-X.

[35] A. Zygmund. Trigonometric Series, volume 1, 2. Cambridge Univ. Press, 1959. 